\title{
COORDINATES AND AUTOMORPHISMS OF POLYNOMIAL AND FREE ASSOCIATIVE ALGEBRAS OF RANK THREE
}

\author{
VESSELIN DRENSKY AND JIE-TAI YU
}

\begin{abstract}
We study $z$-automorphisms of the polynomial algebra $K[x, y, z]$ and the free associative algebra $K\langle x, y, z\rangle$ over a field $K$, i.e., automorphisms which fix the variable $z$. We survey some recent results on such automorphisms and on the corresponding coordinates. For $K\langle x, y, z\rangle$ we include also results about the structure of the $z$-tame automorphisms and algorithms which recognize $z$-tame automorphisms and $z$-tame coordinates.
\end{abstract}

\section{INTRODUCTION}

Let $K$ be an arbitrary field of any characteristic. The automorphism group Aut $K\left[x_{1}, \ldots, x_{n}\right]$ of the polynomial algebra in $n$ variables is well understood only for $n=1$ and $n=2$. The description is trivial for $n=$ 1 , when the elements $\varphi \in$ Aut $K\left[x_{1}\right]$ are defined by $\varphi\left(x_{1}\right)=\alpha x_{1}+\beta$, where $\alpha \in K^{*}=K \backslash 0$ and $\beta \in K$. The classical result of Jung [D] and van der Kulk $\left[\underline{\mathrm{K}}\right.$ gives that all automorphisms of $K\left[x_{1}, x_{2}\right]$ are tame. Writing the automorphisms of $K\left[x_{1}, \ldots, x_{n}\right]$ as $n$-tuples of the images of the variables, and using $x, y$ instead of $x_{1}, x_{2}$, this means that Aut $K[x, y]$ is generated by the affine automorphisms

$$
\psi=\left(\alpha_{11} x+\alpha_{21} y+\beta_{1}, \alpha_{12} x+\alpha_{22} y+\beta_{2}\right), \quad \alpha_{i j}, \beta_{j} \in K,
$$

(and $\psi_{1}=\left(\alpha_{11} x+\alpha_{21} y, \alpha_{12} x+\alpha_{22} y\right)$, the linear part of $\psi$, is invertible) and the triangular automorphisms

$$
\rho=\left(\alpha_{1} x+p_{1}(y), \alpha_{2} y+\beta_{2}\right), \quad \alpha_{1}, \alpha_{2} \in K^{*}, p_{1}(y) \in K[y], \beta_{2} \in K .
$$

2000 Mathematics Subject Classification. Primary 16S10. Secondary 16W20; 16Z05; 13B10; 13B25; 14E07.

Key words and phrases. Automorphisms of free and polynomial algebras, tame automorphisms, wild automorphisms, coordinates, primitive elements in free algebras.

The research of Vesselin Drensky was partially supported by Grant MI-1503/2005 of the Bulgarian National Science Fund.

The research of Jie-Tai Yu was partially supported by a Hong Kong RGC-CERG Grant. 
This result was the starting point of research in several directions, among them the study of the automorphisms of $K\left[x_{1}, \ldots, x_{n}\right]$ for $n>2$, of the automorphisms of the polynomial algebra $A\left[x_{1}, x_{2}\right]$ over an arbitrary commutative ring $A$, and the automorphisms of free associative algebras $K\left\langle x_{1}, \ldots, x_{n}\right\rangle$. In all these cases the tame automorphisms are defined by analogy with the case of $K[x, y]$, as compositions of affine and triangular automorphisms. One studies not only the automorphisms but also the coordinates, i.e., the images of $x_{1}$ under the automorphisms of $K\left[x_{1}, \ldots, x_{n}\right]$.

Nagata [N] constructed the automorphism of $K[x, y, z]$

$$
\nu=\left(x-2\left(y^{2}+x z\right) y-\left(y^{2}+x z\right)^{2} z, y+\left(y^{2}+x z\right) z, z\right)
$$

which fixes $z$. He showed that $\nu$ is nontame, or wild, considered as an automorphism of $K[z][x, y]$, and conjectured that it is wild also as an element of Aut $K[x, y, z]$. This example motivated the study in detail of the automorphisms of $K[z][x, y]$ by several reasons. Their form is simpler than the form of an arbitrary automorphism of $K[x, y, z]$ and one can apply for their study the results on the automorphisms of $K(z)[x, y]$, the polynomial algebra in two variables $x, y$, with rational in $z$ coefficients. Also, the automorphism group of $K[z][x, y]$ provides important examples and conjectures for Aut $K[x, y, z]$. We shall mention only few facts related with the topic of the present paper. It is relatively easy to see (and to decide algorithmically) whether an endomorphism of $K[z][x, y]$ is an automorphism and whether this automorphism is $z$-tame, or tame as an automorphism of $K[z][x, y]$, but a similar problem is much harder for coordinates. When $K$ is a field of characteristic 0, Drensky and Yu DY2] presented a simple algorithm which decides whether a polynomial $f(x, y, z) \in K[x, y, z]$ is a $z$ coordinate and whether this coordinate is $z$-tame. This provided many new wild automorphisms and wild coordinates of $K[z][x, y]$. These results in DY2 are based on a similar algorithm of Shpilrain and Yu SY1] which recognizes the coordinates of $K[x, y]$. The pioneer work of Shestakov and Umirbaev [SU1, SU2, SU3] established that the Nagata automorphism is wild. It also implies that every wild automorphism of $K[z][x, y]$ is wild as an automorphism of $K[x, y, z]$. Umirbaev and $\mathrm{Yu}$ UY] showed that the $z$-wild coordinates in $K[z][x, y]$ are wild also in $K[x, y, z]$. In this way, all $z$-wild examples in DY2 give automatically wild examples in $K[x, y, z]$. 
Going to free algebras, Czerniakiewicz $[\mathrm{Cz}$ and Makar-Limanov [ML1, ML2 proved that all automorphisms of $K\langle x, y\rangle$ are also tame. There are several candidates for wild automorphisms of free algebras with more than two generators. One of them is the example of Anick $(x+(y(x y-y z), y, z+(z y-y z) y) \in$ Aut $K\langle x, y, z\rangle$, see the book by Cohn C2, p. 343. It fixes one variable and its abelianization is a tame automorphism of $K[x, y, z]$. Exchanging the places of $y$ and $z$, we obtain the automorphism $(x+z(x z-z y), y+(x z-z y) z, z)$ which fixes $z$ (or a $z$-automorphism), and refer to it as the Anick automorphism. It is linear in $x$ and $y$, considering $z$ as a "noncommutative constant". Drensky and $\mathrm{Yu}$ [DY3] showed that such $z$-automorphisms are $z$-wild if and only if a suitable invertible $2 \times 2$ matrix with entries from $K\left[z_{1}, z_{2}\right]$ is not a product of elementary matrices. In particular, this gives that the Anick automorphism is $z$-wild. For better understanding of $z$-wild automorphisms one has to know more about $z$-tame automorphisms. The very recent paper by Drensky and Yu [DY7] describes the structure of the group of $z$-tame automorphisms of $K\langle x, y, z\rangle$ and gives algorithms which recognize $z$-tame automorphisms and coordinates of $K\langle x, y, z\rangle$.

When $K$ is a field of characteristic 0, Umirbaev [U2] developed further his ideas with Shestakov and described the defining relations of the group of tame automorphisms of $K[x, y, z]$. As a result, if $\varphi=(f, g, h) \in$ Aut $K\langle x, y, z\rangle$ has the property that the endomorphism $\varphi_{0}=\left(f_{0}, g_{0}, z\right)$ of $K\langle x, y, z\rangle$, where $f_{0}, g_{0}$ are the linear in $x, y$ components of $f, g$, respectively, is a $z$-wild automorphism, then $\varphi$ is wild as an automorphism of $K\langle x, y, z\rangle$. This implies that the Anick automorphism is wild. Finally, we want to mention the recent results of Drensky and Yu [DY4, DY5] which allow to show the wildness of a big class of automorphisms and coordinates of $K\langle x, y, z\rangle$. Many of them cannot be handled with direct application of the methods of DY3] and U2].

In the present paper we give a survey of some, mostly recent results on $z$-automorphisms of $K[x, y, z]$ and $K\langle x, y, z\rangle$. We present also a selection of facts on the automorphisms of $K[x, y]$ and $K\langle x, y\rangle$ as a preparation to the study of the $z$-automorphisms of $K[x, y, z]$ and $K\langle x, y, z\rangle$. (For more details we refer to the books by van den Essen [E2], Mikhalev, Shpilrain, and Yu [MSY], and our survey article DY1.) Finally, we provide a list of open problems and conjectures. 


\section{A SURVEY ON AUTOMORPHISMS OF POLYNOMIAL ALGEBRAS}

We fix the field $K$ and consider the polynomial algebra $K[x, y, z]$ in three variables. We denote its automorphisms as $\varphi=(f, g, h)$, where $f=\varphi(x), g=\varphi(y), h=\varphi(z)$. The multiplication is from right to left. If $\varphi, \psi \in$ Aut $K[x, y, z]$, then in $\varphi \psi$ we first apply $\psi$ and then $\varphi$. Hence, if $\varphi=(f, g, h)$ and $\psi=(u, v, w)$, then

$$
\varphi \psi=(u(f, g, h), v(f, g, h), w(f, g, h)) .
$$

We call the automorphism $\varphi$ a $z$-automorphism if $\varphi(z)=z$, and denote the automorphism group of the $K[z]$-algebra $K[z][x, y]$ by Aut $K[z][x, y]=$ Aut $_{z} K[x, y, z]$. If we want to emphasize that we work with $z$-automorphisms, we write $\varphi=(f, g)$, omitting the third coordinate $z$. The affine and triangular automorphisms of $K[x, y, z]$ are, respectively, of the form $\psi=\left(\alpha_{11} x+\alpha_{21} y+\alpha_{31} z+\beta_{1}, \alpha_{12} x+\alpha_{22} y+\alpha_{32} z+\beta_{2}, \alpha_{13} x+\alpha_{23} y+\alpha_{33} z+\beta_{3}\right)$, $\alpha_{i j}, \beta_{j} \in K$, the $3 \times 3$ matrix $\left(\alpha_{i j}\right)$ being invertible,

$$
\rho=\left(\alpha_{1} x+p_{1}(y, z), \alpha_{2} y+p_{2}(z), \alpha_{3} z+\beta_{3}\right),
$$

$\alpha_{j} \in K^{*}, p_{1} \in K[y, z], p_{2} \in K[z], \beta_{3} \in K$.

We denote by TAut $K[x, y, z]$ the group of the tame automorphisms which is generated by affine and triangular automorphisms. When we consider $z$-automorphisms, the $z$-affine and $z$-triangular automorphisms are, respectively, of the form

$$
\psi=\left(\alpha_{11} x+\alpha_{21} y+b_{1}(z), \alpha_{12} x+\alpha_{22} y+b_{2}(z)\right),
$$

again $\alpha_{i j} \in K, b_{j}(z) \in K[z]$, the $2 \times 2$ matrix $\left(\alpha_{i j}\right)$ being invertible,

$$
\rho=\left(\alpha_{1} x+p_{1}(y, z), \alpha_{2} y+p_{2}(z)\right),
$$

$\alpha_{j} \in K^{*}, p_{1} \in K[y, z], p_{2} \in K[z]$. The group of the $z$-tame automorphisms is TAut $K[z][x, y]=$ TAut $_{z} K[x, y, z]$. We may define the $z$-affine automorphisms by

$$
\psi=\left(a_{11} x+a_{21} y+b_{1}, a_{12} x+a_{22} y+b_{2}\right),
$$

where $a_{i j}(z), b_{j}(z) \in K[z]$ and the $2 \times 2$ matrix $\left(a_{i j}\right)$ is invertible over $K[z]$ (hence its determinant is a nonzero constant in $K$ ). But we shall see that this definition is not convenient in the noncommutative case. For example, the Anick automorphism is affine in this sense but is wild.

We start our survey with the case of two variables accepting notation similar to the case of three variables. Jung [J] in 1942 for $K=\mathbb{C}$ and van der Kulk $[\underline{\mathrm{K}}]$ in 1953 for an arbitrary field $K$ proved that 
Aut $K[x, y]=$ TAut $K[x, y]$. The proof of van der Kulk gives also the structure of Aut $K[x, y]$.

Theorem 1.1. Every automorphism of $K[x, y]$ is tame. The group Aut $K[x, y]$ is isomorphic to the free product $A *_{C} B$ of the group $A$ of the affine automorphisms and the group $B$ of triangular automorphisms amalgamating their intersection $C=A \cap B$.

Every automorphism $\varphi$ can be presented as a product

$$
\varphi=\psi_{m}^{\varepsilon_{m}} \rho_{m} \psi_{m-1} \cdots \rho_{2} \psi_{1} \rho_{1}^{\varepsilon_{1}}
$$

where $\psi_{i} \in A, \rho_{i} \in B\left(\varepsilon_{1}\right.$ and $\varepsilon_{m}$ are equal to 0 or 1$)$, and, if $\varphi$ does not belong to the union of $A$ and $B$, we may assume that $\psi_{i} \in$ $A \backslash B, \rho_{i} \in B \backslash A$. The freedom of the product means that if $\varphi$ has a nontrivial presentation of this form, then it is different from the identity automorphism. Fixing the linear nontriangular automorphism $\tau=(y, x)$, we can present $\varphi$ in the canonical form

$$
\varphi=\rho_{n} \tau \cdots \tau \rho_{1} \tau \rho_{0},
$$

where $\rho_{0}, \rho_{1}, \ldots, \rho_{n} \in B$ and only $\rho_{0}$ and $\rho_{n}$ are allowed to belong to $A$, see for example p. 350 in C2 . Using the equalities for compositions of automorphisms

$$
\begin{gathered}
(\alpha x+p(y), \beta y+\gamma)=\left(x+\alpha^{-1}(p(y)-p(0)), y\right)(\alpha x+p(0), \beta y+\gamma), \quad \gamma \in K, \\
(\alpha x+\xi, \beta y+\eta) \tau=(\beta y+\eta, \alpha x+\xi)=\tau(\beta x+\eta, \alpha y+\xi), \quad \xi, \eta \in K,
\end{gathered}
$$

we can do further simplifications in (11), assuming that $\rho_{i}=\left(x+p_{i}(y), y\right)$ with $p_{i}(0)=0$ for all $i=1, \ldots, n$. We also assume that $\rho_{0}=\left(\alpha_{0} x+\right.$ $\left.p_{0}(y), \beta_{0} y+\gamma_{0}\right)$.

Let $\varphi=(f, g)$ and let us assume for example that $\operatorname{deg} p_{i}(y)=d_{i}>1$ for all $i=0,1, \ldots, n$, and let $p_{i}(y)=\xi_{i} y^{d_{i}}+\cdots$, where $\xi_{i} \in K^{*}$ and $q_{i}(y)=\overline{p_{i}}(y)=\xi_{i} y^{d_{i}}$ is the leading monomial of $p_{i}(y)$. Direct computations show that deg $f=d_{0} \cdot \operatorname{deg} g$ and the monomials of highest degree of $f, g$ are, respectively,

$$
\begin{gathered}
\bar{f}=q_{0}\left(q_{1}\left(\ldots\left(q_{n}(y)\right) \ldots\right)\right)=\xi_{0}\left(\zeta y^{N}\right)^{d_{0}}, \\
\bar{g}=\beta_{0} q_{1}\left(\ldots\left(q_{n}(y)\right) \ldots\right)=\beta_{0} \zeta y^{N} .
\end{gathered}
$$

Hence, up to a multiplicative constant, $\bar{f}$ is equal to a power of $\bar{g}$ and the monomial $q_{0}(y)$ can be determined uniquely from $\bar{f}, \bar{g}$. Similar relations hold in the cases when some of the triangular automorphisms $\rho_{0}$ and $\rho_{n}$ is affine but $\bar{f}, \bar{g}$ may be powers of a linear combination of $x, y$ instead of powers of $y$. These considerations provide an easy algorithm 
to decide whether an endomorphism of $K[x, y]$ is an automorphism, see Theorem 6.8.5 in [C2]. Of course, in all algorithms we assume that the field $K$ is constructive.

Algorithm 1.2. Let $\varphi=(f, g)$ be an endomorphism of $K[x, y]$.

Step 0. If some of the polynomials $f, g$ is a constant from $K$, then $\varphi$ is not an automorphism.

Step 1. Let $u, v$ be the homogeneous components of highest degree of $f, g$, respectively. If both $u, v$ are linear, then we check whether they are linearly independent. If yes, then $\varphi$ is an affine automorphism. If $u, v$ are linearly dependent, then $\varphi$ is not an automorphism.

Step 2. If $u$ is not linear, $\operatorname{deg} u \geq \operatorname{deg} v$, and $u=\beta v^{d}$ for some $\beta \in$ $K^{*}$ and some $d \geq 1$, then we replace $\varphi=(f, g)$ with $\varphi_{1}=\left(f-\beta g^{d}, g\right)$. Then we apply Step 0 to $\varphi_{1}$. If $u$ cannot be presented in the form $u=\beta v^{d}$, then $\varphi$ is not an automorphism.

Step 3. If $v$ is not linear, $\operatorname{deg} u<\operatorname{deg} v$, and $v=\beta u^{d}$, then we replace $\varphi=(f, g)$ with $\varphi_{1}=\left(f, g-\beta f^{d}\right)$. Then we apply Step 0 to $\varphi_{1}$. If $v$ cannot be presented in the form $v=\beta u^{d}$, then $\varphi$ is not an automorphism.

Steps 2 and 3 of the algorithm correspond to a triangular automorphism and the algorithm also provides a decomposition of $\varphi$ as a product of affine and triangular automorphisms. If $\varphi_{1}=\left(f-\beta g^{d}, g\right)$ in Step 2, and $\sigma_{1}=\left(x-\beta y^{d}, y\right)$, then $\varphi_{1}=\varphi \sigma_{1}$ and $\varphi=\varphi_{1} \sigma_{1}^{-1}$. If $\varphi_{1}=\left(f, g-\beta f^{d}\right)$ in Step 3, then $\varphi_{1}=\varphi \tau \sigma_{1} \tau$ and $\varphi=\varphi_{1} \tau \sigma_{1}^{-1} \tau$.

In the general case of polynomial algebras in several variables, there is an effective algorithm which decides whether an endomorphism is an automorphism. It involves Gröbner bases techniques, see our survey article DY1] for references.

Algorithm 1.2 can be modified to decide whether a polynomial $f(x, y)$ is a coordinate of $K[x, y]$. We start with the analysis of the behavior of the first coordinate $f$ of $\varphi$ in (11). Note, that if $\varphi=(f, g)$ and $\varphi^{\prime}=\left(f, g^{\prime}\right)$ are two automorphisms with the same first coordinate, then $\varphi^{-1} \varphi^{\prime}$ fixes $x$. Hence $\varphi^{-1} \varphi^{\prime}=\left(x, g^{\prime \prime}\right)$ and, obligatorily, $g^{\prime \prime}=\beta y+r(x)$. In this way, if we find one "coordinate mate" $g$ of $f$, then we can find all other mates. Let $(a, b)$ be a pair of positive integers. We define the $(a, b)$-degree of a monomial $x^{m} y^{n}$ as $\operatorname{deg}_{(a, b)} x^{m} y^{n}=a m+b n$, and denote the homogeneous component of maximal $(a, b)$-degree of $f \in K[x, y]$ by $|f|_{(a, b)}$. Let us assume again that $\operatorname{deg} p_{i}(y)>1$ for all $i=0,1, \ldots, n$, 
and let $h$ be the first coordinate of $\psi=\rho_{n-1} \tau \cdots \tau \rho_{1} \tau \rho_{0}$. Then

$$
\bar{h}=q_{0}\left(q_{1}\left(\ldots\left(q_{n-1}(y)\right) \ldots\right)\right)=\vartheta y^{M}, \quad \vartheta \in K^{*}, M \geq 2 .
$$

The homogeneous component of maximal $\left(d_{n}, 1\right)$-degree of $x+p_{n}(y)$ is $x+\xi_{n} y^{d_{n}}$ and direct calculations give

$$
|f|_{\left(d_{n}, 1\right)}=\left|\rho_{n} \tau(\bar{h})\right|_{\left(d_{n}, 1\right)}=\vartheta\left(x+\xi_{n} y^{d_{n}}\right)^{M} .
$$

Hence we can recover from here $d_{n}$ and $\xi_{n}$. In particular,

$$
f=\omega\left(x^{M}+\xi_{n}^{M} y^{M d_{n}}\right)+\cdots .
$$

The considerations are similar when some of the automorphisms $\rho_{0}$ and $\rho_{n}$ is affine. We easily obtain the validity of the following algorithm which recognizes the coordinates in $K[x, y]$.

Algorithm 1.3. Let $f(x, y) \in K[x, y]$ be a polynomial.

Step 0. If $f$ is a constant from $K$, then $f$ is not a coordinate. If $f=\lambda_{1} x+\lambda_{2} y+\lambda_{3}$ is linear, then $f$ is a coordinate. One of its mates is of the form $g=\mu_{1} x+\mu_{2} y+\mu_{3}$ and can be determined from the property that the polynomials $\lambda_{1} x+\lambda_{2} y$ and $\mu_{1} x+\mu_{2} y$ are linearly independent.

Step 1. If $\operatorname{deg} f>1$ and $f$ depends only on $x$ (or only on $y$ ), then it is not a coordinate. If $f$ does not contain as a summand $\eta x^{M}$ or $\zeta y^{N}$ for some $\eta, \zeta \in K^{*}$ and $M, N \geq 1$, then $f$ is not a coordinate.

Step 2. Let $f=\eta x^{M}+\zeta y^{N}+\cdots$, where $\cdots$ stays for the linear combination of the monomials of the form $x^{j}, j<M, y^{k}, k<N$, and the monomials $x^{r} y^{s}, r, s \geq 1$. If $M$ does not divide $N$ and $N$ does not divide $M$, then $f$ is not a coordinate.

Step 3. Let $M$ divide $N$ and $N=M d$. Write $\zeta$ in the form $\zeta=\eta \xi^{d}$, $\xi \in K^{*}$. (If $K$ is not algebraically closed and this is not possible, then $f$ is not a coordinate of $K[x, y]$.) Consider the $(d, 1)$-grading of $K[x, y]$. If $|f|_{(d, 1)} \neq \eta\left(x+\xi y^{d}\right)^{M}$, then $f$ is not a coordinate. If $|f|_{(d, 1)}=$ $\eta\left(x+\xi y^{d}\right)^{M}$ and $M>1$, then define $\vartheta=\left(x+\xi y^{d}, y\right)$, replace $f$ with $f_{1}=\vartheta^{-1}(f)$ and go to Step 0. If $M=1$ and $|f|_{(d, 1)}=\eta\left(x+\xi y^{d}\right)$, then $u=f-\eta\left(x+\xi y^{d}\right)$ does not depend on $x$ and hence $f=\eta x+\xi y^{d}+u(y)$ is a coordinate. As a mate, we can take $g=y$.

Step 4. Let $N$ divide $M$ and $M=N d, d>1$. The considerations are similar to those of Step 3, working with $\vartheta=\left(x, y+\xi x^{d}\right)$ instead of with $\vartheta=\left(x+\xi y^{d}, y\right)$.

As in the previous algorithm, Steps 3 and 4 correspond to a triangular automorphism. For example, if $|f|_{(d, 1)}=\eta\left(x+\xi y^{d}\right)^{M}$ and $M>1$ 
in Step 3, and $u=f-\eta\left(x+\xi y^{d}\right)^{M}$, then $\vartheta^{-1}(f)=\vartheta^{-1}(u)+\eta x^{M}$. Since $x+\xi y^{d}$ and $y$ are $(d, 1)$-homogeneous, the $(d, 1)$-degree of $\vartheta^{-1}(u)$ is smaller than this of $f$. Hence $\vartheta^{-1}(f)$ does not contain summands of the form $\zeta_{j} y^{j}, j \geq M d=N$ and we decrease the sum $M+N$.

To the best of our knowledge, the above algorithm had not been explicitly stated before [SY3], where Shpilrain and Yu established an algorithm which gives a canonical form, up to automorphic equivalence, of a class of polynomials in $K[x, y]$. The automorphic equivalence problem for $K[x, y]$ asks how to decide whether, for two given polynomials $p, q \in K[x, y]$, there exists an automorphism $\varphi$ such that $q=\varphi(p)$. It was solved over $\mathbb{C}$ by Wightwick Wi] and, over an arbitrary algebraically closed constructive field $K$, by Makar-Limanov, Shpilrain, and $\mathrm{Yu}$ [MLSY].

For a polynomial $f(x, y) \in K[x, y]$ we denote by

$$
f_{x}=\frac{\partial f}{\partial x}, \quad f_{y}=\frac{\partial f}{\partial y}
$$

the partial derivatives of $f$ with respect to $x$ and $y$. If $\varphi=(f, g)$ is an endomorphism of $K[x, y]$, then its Jacobian matrix is

$$
J(\varphi)=\left|\begin{array}{ll}
f_{x} & g_{x} \\
f_{y} & g_{y}
\end{array}\right| .
$$

For polynomial algebras in more than two variables the Jacobian matrix is defined in a similar way. The chain rule gives that

$$
J(\varphi \psi)=J(\varphi) \varphi(J(\psi)),
$$

where $\varphi$ acts on $J(\psi)$ componentwise. This implies that if $\varphi$ is an automorphism, then its Jacobian matrix $J(\varphi)$ is invertible and the determinant $\operatorname{det} J(\varphi)$ is a nonzero scalar in $K$. The famous Jacobian conjecture states that if char $K=0$ and $J(\varphi)$ is invertible, then the endomorphism $\varphi$ is an automorphism.

If $\rho$ is a triangular automorphism, then its Jacobian matrix $J(\rho)$ is a low triangular matrix. If $\psi$ is an affine automorphism, then $J(\psi)$ belongs to $G L_{2}(K)$ and also is a product of low and upper triangular matrices. Since the automorphisms of $K[x, y]$ are tame, the chain rule gives that $J(\varphi)$ is a product of triangular matrices for all $\varphi \in$ Aut $K[x, y]$. Hence $J(\varphi)$ belongs to the group $G E_{2}(K[x, y])$ generated by the elementary $2 \times 2$ matrices with entries from $K[x, y]$ and diagonal matrices. This fact was used by Wright $\mathrm{Wr}$ to solve the Jacobian conjecture for endomorphisms $\varphi$ of $K[x, y]$ with the property 
$J(\varphi) \in G E_{2}(K[x, y])$. Wright $\mathrm{Wr}$ established also that the group $G E_{2}\left(K\left[x_{1}, \ldots, x_{n}\right]\right)$ is a free product of $G L_{2}(K)$ and the group of low triangular matrices with entries from $K\left[x_{1}, \ldots, x_{n}\right]$ with amalgamation of their intersection. Hence $G E_{2}\left(K\left[x_{1}, \ldots, x_{n}\right]\right)$ has a similar structure as Aut $K[x, y]$. There is an algorithm which decides whether a matrix in $G L_{2}\left(K\left[x_{1}, \ldots, x_{n}\right]\right)$ belongs to $G E_{2}\left(K\left[x_{1}, \ldots, x_{n}\right]\right)$. It was suggested by Tolhuizen, Hollmann and Kalker [THK] for the partial ordering by degree and then generalized by Park $[\mathrm{P}]$ for any term-ordering on $K\left[x_{1}, \ldots, x_{n}\right]$. One applies Gaussian elimination process on the matrix based on the Euclidean division algorithm for $K\left[x_{1}, \ldots, x_{n}\right]$. The matrix belongs to $G E_{2}\left(K\left[x_{1}, \ldots, x_{n}\right]\right)$ if and only if this procedure brings it to an elementary or diagonal matrix. The idea of Wright $\mathrm{Wr}$ and this algorithm were combined by Shpilrain and Yu [SY1] to give a very simple algorithm which decides whether a polynomial $f(x, y) \in K[x, y]$, char $K=0$, is a coordinate.

Let $K$ be a constructive field of characteristic 0 . We fix a termordering on $K[x, y]$. Recall that this a linear order on the set of monomials $x^{m} y^{n}$, which is a multiplicatively compatible well-ordering.

Algorithm 1.4. (Shpilrain and Yu SYY] Let $f(x, y) \in K[x, y]$. Consider the partial derivatives $p=f_{x}$ and $q=f_{y}$.

Step 0. If $p=q=0$, then $f$ is not a coordinate. If either $p=0$ and $q \in K^{*}$, or $q=0$ and $p \in K^{*}$ then $f$ is a coordinate. If either $p=0$ and $\operatorname{deg} q>0$, or $q=0$ and $\operatorname{deg} p>0$ then $f$ is not a coordinate.

Step 1. If $p, q \neq 0$, apply the Euclidean division algorithm to $p$ and $q$. If this is impossible, then $f$ is not a coordinate. If $p=q s+r$, where $r, s \in K[x, y]$ with $r=0$ or $\operatorname{deg} r<\operatorname{deg} q$, then replace $p$ with $r$ and go to Step 0. If $q=p s+r$, where $r, s \in K[x, y]$ with $r=0$ or $\operatorname{deg} r<\operatorname{deg} p$, then replace $q$ with $r$ and again go to Step 0 .

Before [SY1, SY3, more complicated algorithms which decide whether $f(x, y) \in \mathbb{C}[x, y]$ is a coordinate were given by Chądzyński and Krasiński CK in terms of the so called Łojasiewicz exponent at infinity and by van den Essen [E1] using the technique of locally nilpotent derivations.

Now we continue our survey with exposition on automorphisms of $K[z][x, y]$ and $K[x, y, z]$.

The first example of a $z$-wild automorphism of $K[z][x, y]$ was constructed by Nagata [N].

Theorem 1.5. (Theorem 1.4, p. 42 [N]) The Nagata automorphism

$$
\nu=\left(x-2 y\left(y^{2}+x z\right)-z\left(y^{2}+x z\right)^{2}, y+z\left(y^{2}+x z\right)\right)
$$


of $K[z][x, y]$ is z-wild.

Conjecture 1.6. The Nagata automorphism is wild as an automorphism of the polynomial algebra $K[x, y, z]$.

The Nagata automorphism is among the most investigated automorphisms of $K[x, y, z]$. It was used as a counterexample to different problems on automorphisms of polynomial algebras and group actions on affine spaces, see e.g. the paper by Bass [Ba and our survey [DY1]. We shall consider only one property of the Nagata automorphism.

Recall that a $K$-linear operator $\delta$ acting on $K\left[x_{1}, \ldots, x_{n}\right]$ is a derivation, if $\delta(u v)=\delta(u) v+u \delta(v)$ for all $u, v \in K\left[x_{1}, \ldots, x_{n}\right]$. The derivation $\delta$ is triangular if $\delta\left(x_{j}\right) \in K\left[x_{j+1}, \ldots, x_{n}\right], j=1, \ldots, n$. The derivation $\delta$ is locally nilpotent if for every $u$ there exists a $d$ such that $\delta^{d}(u)=0$. If $\delta$ is locally nilpotent and char $K=0$, then the formal series

$$
\exp (\delta)=1+\frac{\delta}{1 !}+\frac{\delta^{2}}{2 !}+\cdots
$$

is a well defined linear operator on $K\left[x_{1}, \ldots, x_{n}\right]$ which is an automorphism of the polynomial algebra. Clearly, all triangular derivations are locally nilpotent. The corresponding exponential automorphisms are also triangular and hence tame. Another way to construct locally nilpotent derivations is to start with a locally nilpotent derivation $\delta$ and to consider its kernel $\operatorname{Ker} \delta$. If $0 \neq w \in \operatorname{Ker} \delta$, then $\Delta=w \delta$ is also a locally nilpotent derivation with the same kernel.

Example 1.7. Let char $K=0$ and let $\delta$ be the triangular derivation of $K[x, y, z]$ defined by

$$
\delta(x)=-2 y, \quad \delta(y)=z, \quad \delta(z)=0 .
$$

Then Ker $\delta=K\left[y^{2}+x z, z\right]$. For $w=y^{2}+x z$ and $\Delta=w \delta$ we obtain that $\nu=\exp (\Delta)$, the Nagata automorphism, because

$$
\begin{gathered}
\exp (\Delta): x \rightarrow\left(1+\frac{\Delta}{1 !}+\frac{\Delta^{2}}{2 !}+\cdots\right)(x) \\
=x+\left(y^{2}+x z\right) \frac{\delta(x)}{1 !}+\left(y^{2}+x z\right)^{2} \frac{\delta^{2}(x)}{2 !}+\cdots=x-2\left(y^{2}+x z\right) y-\left(y^{2}+x z\right)^{2} z \\
\exp (\Delta): y \rightarrow\left(1+\frac{\Delta}{1 !}+\frac{\Delta^{2}}{2 !}+\cdots\right)(y) \\
=y+\left(y^{2}+x z\right) \frac{\delta(y)}{1 !}+\cdots=y+\left(y^{2}+x z\right) z
\end{gathered}
$$




$$
\exp (\Delta): z \rightarrow\left(1+\frac{\Delta}{1 !}+\frac{\Delta^{2}}{2 !}+\cdots\right)(z)=z
$$

The automorphism $\varphi$ of the polynomial algebra $K\left[x_{1}, \ldots, x_{n}\right]$ is called stably tame, if it becomes tame when extended identically, by $\varphi\left(x_{n+i}\right)=x_{n+i}, i=1, \ldots, m$, on the polynomial algebra $K\left[x_{1}, \ldots, x_{n+m}\right]$ for a suitable integer $m$. The following theorem of Smith SSm gives a big class of stably tame automorphisms.

Theorem 1.8. Over a field $K$ of characteristic 0, every triangular derivation $\delta$ of $K\left[x_{1}, \ldots, x_{n}\right]$ and every $w \in \operatorname{Ker} \delta$, the automorphism $\exp (w \delta)$ is stably tame and becomes tame on $K\left[x_{1}, \ldots, x_{n}, x_{n+1}\right]$ assuming that $\delta\left(x_{n+1}\right)=0$.

The key observation in the proof of the theorem is the following equation in $K\left[x_{1}, \ldots, x_{n}, x_{n+1}\right]$ discovered by Smith

$$
\exp (w \delta)=\vartheta^{-1} \cdot \exp \left(x_{n+1} \delta\right) \cdot \vartheta \cdot \exp ^{-1}\left(x_{n+1} \delta\right),
$$

where $\vartheta=\left(x_{1}, \ldots, x_{n}, x_{n+1}+w\right)$. Since $w \in K\left[x_{1}, \ldots, x_{n}\right]$, the automorphism $\vartheta$ is tame (triangular assuming that $x_{n+1}<x_{i}, i=1, \ldots, n$ ), the automorphism $\exp \left(x_{n+1} \delta\right)$ is also tame because $\delta$ is a triangular derivation (and $\exp \left(x_{n+1} \delta\right)$ is a triangular automorphism assuming that $\left.x_{i}<x_{n+1}\right)$. Hence, $\exp (w \delta)$ is also tame as a composition of tame automorphisms of $K\left[x_{1}, \ldots, x_{n}, x_{n+1}\right]$.

The approach of Smith is one of the main steps in the proofs of all known results on stable tameness of automorphisms of polynomial algebras. Recently, the notion of stable tameness was transferred also to coordinates, see Berson [B1, B2]. The coordinate $f \in K\left[x_{1}, \ldots, x_{n}\right]$ is called stably tame, if, for some $m$, there exists a tame automorphism $\varphi$ of $K\left[x_{1}, \ldots, x_{n+m}\right]$ such that $\varphi\left(x_{1}\right)=f$. This tameness is weaker than the tameness of automorphisms because one does not require that $\varphi$ fixes the variables $x_{n+1}, \ldots, x_{n+m}$. Berson [B2] showed that, over a noetherian $\mathbb{Q}$-domain $A$ of Krull dimension one, all coordinates of $A[x, y]$ are stably tame. Hence every $z$-coordinate of $K[z][x, y]$ is stably tame. For a class of coordinates of $A[x, y]$ Edo [Ed] proved that they are coordinates of stably tame automorphisms. We shall mention the following well known problem.

Problem 1.9. Are all automorphisms of $K[z][x, y]$ stably tame?

Every $z$-automorphism of $K[z][x, y]$ is also an automorphism of the polynomial algebra $K(z)[x, y]$ over the field of fractions $K(z)$. Hence 
one may use the theory of automorphisms over a field to study zautomorphisms. Let us consider the chain of inclusions

TAut $K[z][x, y] \subset$ Aut $K[z][x, y] \subset$ Aut $K(z)[x, y]$

and the equality

$$
\text { Aut } K(z)[x, y]=A(K(z)[x, y]) *_{C(K(z)[x, y])} B(K(z)[x, y]),
$$

where $A(K(z)[x, y]), B(K(z)[x, y])$, and $C(K(z)[x, y])$ are, respectively, the groups of affine and triangular automorphisms of $K(z)[x, y]$, and the intersection of these two groups. The nice structure of Aut $K(z)[x, y]$ should imply some structure on TAut $K[z][x, y]$ and on Aut $K[z][x, y]$. For any commutative ring $A$ we denote by $\operatorname{Aut}^{0} A[x, y]$ the subgroup of Aut $A[x, y]$ consisting of all automorphisms $\varphi=(f, g)$ with the property $f(0,0)=g(0,0)=0$. We use similar notation for other groups of automorphisms. We call the elements of $\operatorname{Aut}^{0} A[x, y]$ automorphisms without constant terms. For many purposes the study of $\operatorname{Aut}^{0} A[x, y]$ is equivalent to that of Aut $A[x, y]$ because every automorphism of $A[x, y]$ is a composition of a translation $(x+\alpha, y+\beta)$ and an automorphism without constant terms. Although the following result of Wright $\mathrm{Wr}$ holds in a more general situation, we shall state it in the case of $K[z][x, y]$ only.

Theorem 1.10. (i) Over any field $K$ the group TAut $K[z][x, y]$ and its subgroup TAut $^{0} K[z][x, y]$ have the amalgamated free product structure

TAut $K[z][x, y]=A *_{C} B, \quad \operatorname{TAut}^{0} K[z][x, y]=G L_{2}(K) *_{C^{0}} B^{0}$, where $A$ and $B$ are, respectively, the group of affine z-automorphisms and the group of triangular z-automorphisms and $C=A \cap B$.

(ii) There is a subgroup $W$ of Aut $^{0} K[z][x, y]$ containing the group $B^{0}(K[z][x, y])$ of all triangular automorphisms without constant terms such that

$$
G L_{2}(K[z]) \cap W=G L_{2}(K[z]) \cap B^{0}(K[z][x, y])=C^{0}(K[z][x, y]),
$$

and

$$
\operatorname{Aut}^{0} K[z][x, y] \cong G L_{2}(K[z]) *_{C^{0}(K[z][x, y])} W
$$

the amalgamated free product of $G L_{2}(K[z])$ and $W$.

The original statement of Theorem 1.10 involves affine and linear automorphisms with coefficients from $K[z]$ but this is not essential because every invertible matrix with entries in $K[z]$ is a product of elementary and diagonal matrices. 
Theorem 1.10 allows to obtain immediately analogues of Algorithms 1.2 and 1.3 which recognize, respectively, the $z$-tame automorphisms and coordinates, and find the decomposition of the automorphism as a product of affine and triangular automorphisms. Algorithm 1.4 also has its analogue for $z$-tame coordinates of $K[z][x, y]$.

Proposition 1.11. (Drensky and Yu DY2]) Let $K$ be a constructive field of characteristic 0 and let $f(x, y, z) \in K[x, y, z]$. Consider the partial derivatives $p=f_{x}$ and $q=f_{y}$. Then the procedure described in Algorithm 1.4 decides whether $f$ is a $z$-tame coordinate of $K[z][x, y]$.

Of course, not every coordinate of $K(z)[x, y]$ which is in $K[z][x, y]$ is a coordinate also in $K[z][x, y]$. Now we shall describe an algorithm which handles this problem. Recall that $f \in K[z][x, y]$ has a unimodular gradient with respect to $x$ and $y$ if the partial derivatives $f_{x}$ and $f_{y}$ generate $K[z][x, y]$ as an ideal. The following result is obtained by Drensky and Yu [DY2]. Their proof uses a result of Daigle and Freudenburg $[\mathrm{DF}$ on locally nilpotent derivations. The same proof works in the general case, over an arbitrary commutative $\mathbb{Q}$-algebra $A$, see Edo and Vénéreau [EdV].

Theorem 1.12. (Drensky and Yu [DY2]) The polynomial $f(x, y) \in$ $K[z][x, y]$ is a coordinate in $K[z][x, y]$ if and only if it is with unimodular gradient in $K[z][x, y]$ and is a coordinate in $K(z)[x, y]$.

Combined with Algorithm 1.4. Theorem 1.12 allows to solve effectively the problem whether a polynomial is a coordinate, but this time we have to apply Gröbner bases techniques instead of the Euclidean algorithm.

Algorithm 1.13. (Drensky and Yu [DY2]) Given a polynomial $f \in$ $K[z][x, y]$, where $K$ is a constructive field of characteristic 0 , we want to decide whether it is a coordinate.

Step 0. Take the partial derivatives $f_{x}, f_{y} \in K[z][x, y]$.

Step 1. Fix some term-ordering in $K[x, y, z]$. Find the reduced Gröbner basis of the ideal of $K[x, y, z]$ generated by the polynomials $f_{x}, f_{y}$. This ideal coincides with $K[x, y, z]$ if and only if the obtained reduced Gröbner basis consists of a nonzero constant in $K$ only. Hence, if the Gröbner basis does not consist of a nonzero constant, then $f$ is not a coordinate polynomial of $K[z][x, y]$.

Step 2. Working on $K(z)$ instead on $K[z]$ (and fixing a term-ordering in $K[z][x, y]$ ), we apply Algorithm 1.4 and determine whether $f$ is a 
coordinate in $K(z)[x, y]$. If the answer is negative, then $f$ cannot be a coordinate in $K[z][x, y]$. Otherwise $f$ is a coordinate of $K[z][x, y]$.

For example, if $f=y+\left(y^{2}+x z\right) z$ is the second coordinate of the Nagata automorphism, then $f_{x}=z^{2}, f_{y}=1+2 y z$. It is easy to see that $f_{x}$ and $f_{y}$ generate as an ideal the whole $K[x, y, z]$. (For the proof, $1=-4 y^{2} f_{x}+(1-2 y z) f_{y}$ belongs to the ideal generated by $f_{x}$ and $f_{y}$.) Applying the Euclidean algorithm in $K(z)[x, y]$, we see that $f_{y}$ is divisible by $f_{x}$, and $f_{x}$ is a constant, as an element of $K(z)$. Hence $f$ is a coordinate both in $K(z)[x, y]$ and $K[z][x, y]$. Since the leading monomials $z^{2}$ and $2 y z$ of $f_{x}$ and $f_{y}$ are not divisible by each other, we obtain that $f$ is a $z$-wild coordinate.

See also our survey [DY1 and the paper by Berson and van den Essen $[\mathrm{BE}$ for the case of coordinates in $A[x, y]$ over any finitely generated $\mathbb{Q}$-algebra $A$. Going back to the automorphisms of $K[x, y, z]$, Theorem 1.12 and Algorithm 1.13 allow to find effectively a lot of wild automorphisms of $K[z][x, y]$, giving in this way new candidates for wild automorphisms of $K[x, y, z]$, all of them fixing $z$ as in the example suggested by Nagata, see DY1, DY2. One of the possible ways to search for new $z$-wild automorphisms is to try to find triangular automorphisms $\rho_{0}, \rho_{1}, \ldots, \rho_{n}$ of $K(z)[x, y]$ such that $\rho_{0}$ and $\rho_{n}$ are not automorphisms of $K[z][x, y]$ but, nevertheless, $\varphi=\rho_{n} \tau \cdots \tau \rho_{1} \tau \rho_{0}$ in the canonical form (11) belongs to Aut $K[z][x, y]$ (and not to TAut $K[z][x, y]$ ). Such a search was performed for small $n$. For example, the Nagata automorphism, considered as an automorphism of $K(z)[x, y]$ has the presentation

$$
\nu=\rho_{0} \rho_{1} \rho_{0}^{-1}, \quad \text { where } \quad \rho_{0}=\left(x+\frac{y^{2}}{z}, y\right), \quad \rho_{1}=\left(x, y+z^{2} x\right) .
$$

There are also other ways to construct automorphisms of polynomial algebras. One of them uses the algebra $R_{22}$ generated by two generic $2 \times 2$ matrices

$$
X=\left(\begin{array}{ll}
x_{11} & x_{12} \\
x_{21} & x_{22}
\end{array}\right), \quad Y=\left(\begin{array}{ll}
y_{11} & y_{12} \\
y_{21} & y_{22}
\end{array}\right),
$$

where $x_{i j}, y_{i j}$ are commuting variables. Let $T_{22}$ be the noncommutative trace algebra generated by $R_{22}$ and all traces $\operatorname{tr}(u), u \in R_{22}$. If char $K \neq 2$, then the center $C\left(T_{22}\right)$ of $T_{22}$ is isomorphic to the polynomial algebra generated by the five algebraically independent polynomials

$$
\operatorname{tr}(X), \operatorname{tr}(Y), \operatorname{tr}\left(X^{2}\right), \operatorname{tr}(X Y), \operatorname{tr}\left(Y^{2}\right)
$$


Hence every automorphism of the generic matrix algebra $R_{22}$ induces automorphisms on $T_{22}$ and $C\left(T_{22}\right) \cong K\left[x_{1}, \ldots, x_{5}\right]$. This construction was used to obtain different automorphisms of $K\left[x_{1}, \ldots, x_{5}\right]$, see Bergman [Be, Alev and Le Bruyn [AL], Drensky and Gupta DG1, DG2. Nevertheless, it has turned out that all these automorphisms can be described in terms of locally nilpotent derivations, and as naturally looking automorphisms of $K\left(y_{1}, \ldots, y_{m}\right)\left[x_{1}, \ldots, x_{n}\right]$. Compare, for example, the results of [DG1] with those of Drensky, van den Essen, and Stefanov DES.

Till the end of the section we assume that the field $K$ is of characteristic 0. Shestakov and Umirbaev [SU1, SU2, SU3] developed a special technique and established, more than 30 years after Nagata discovered his automorphism, that the Nagata automorphism is wild, as an automorphism of $K[x, y, z]$. A popular exposition of the ideas of their proof is given by van den Essen E3. We shall state some of the results in SU1, SU2, [SU3 and shall say a couple of words for the (very rough) idea of the proofs.

Theorem 1.14. (Shestakov and Umirbaev [SU1, SU2, SU3]) Over a constructive field $K$ of characteristic 0 , there exists an algorithm which decides whether an automorphism of $K[x, y, z]$ is tame.

If $\varphi=(f, g)$ is an automorphism of the polynomial algebra in two variables, which by Theorem 1.1 is tame, then Algorithm 1.2 allows to find a triangular or a linear automorphism which, applied to $f$ and $g$, decreases the sum of their degrees. Hence, we may find a sequence $\psi_{1}, \ldots, \psi_{n}$ of affine and triangular automorphisms such that, for $\varphi_{i}=\psi_{i} \cdots \psi_{1} \varphi$ we have $\operatorname{deg}\left(\varphi_{i+1}(x)\right)+\operatorname{deg}\left(\varphi_{i+1}(y)\right)<\operatorname{deg}\left(\varphi_{i}(x)\right)+$ $\operatorname{deg}\left(\varphi_{i}(y)\right)$. But in the case of $K[x, y, z]$ it may happen that this is not possible. The main idea of the proof of Shestakov and Umirbaev is to use a peak reduction. If $\varphi=(f, g, h)$ is a tame automorphism of $K[x, y, z]$, then one follows the sum $\operatorname{deg} f+\operatorname{deg} g+\operatorname{deg} h$ and tries to minimize it. Let $\bar{f}$ be the homogenous component of maximal degree of $f$. If one of the polynomials $\bar{f}, \bar{g}, \bar{h}$ belongs to the subalgebra of $K[x, y, z]$ generated by the other two, say $\bar{f}=p(\bar{g}, \bar{h})$, then one can reduce the degree immediately replacing $\varphi$ with the tame automorphism $\varphi(x-p(y, z), y, z)$. If neither of the polynomials $\bar{f}, \bar{g}, \bar{h}$ belongs to the subalgebra generated by the others, then the degree cannot be decreased immediately. Nevertheless, depending on the type of the 
triple $(\bar{f}, \bar{g}, \bar{h})$ the authors find a sequence of several affine and triangular automorphisms which decreases the degree. For this purpose, they need a detailed information on the two-generated subalgebras of $K[x, y, z]$ and lower estimates for the degree of the elements of these subalgebras. This is achieved by embedding the polynomial algebra in the free Poisson algebra (or the algebra of universal Poisson brackets) and the usage systematically the brackets as an additional tool. So, the proof involves essentially methods of noncommutative (and even nonassociative) algebra.

Applying the algorithm of Theorem 1.14 Shestakov and Umirbaev solved into affirmative the Nagata conjecture.

Theorem 1.15. (Shestakov and Umirbaev [SU1, SU2, SU3]) The $\mathrm{Na-}$ gata automorphism is wild as an automorphism of $K[x, y, z]$.

They also obtained the following result.

Theorem 1.16. Every z-automorphism of $K[x, y, z]$ which is z-wild is also wild as an automorphism of $K[x, y, z]$.

This theorem replaces the difficult problem to decide whether a $z$ automorphism is wild as an automorphism of $K[x, y, z]$ with the easier one whether it is $z$-wild. Now, one uses the results of Drensky and $\mathrm{Yu}$ DY2 to find new examples of wild automorphisms and to determine whether a z-automorphism is wild.

Following Umirbaev and $\mathrm{Yu}[\mathrm{UY}$, a coordinate $f$ in $K[x, y, z]$ is called wild, if every automorphism $\varphi=(f, g, h)$ having $f$ as a first coordinate is wild. Umirbaev and $\mathrm{Yu}$ proved the following theorem which solves the Strong Nagata Conjecture.

Theorem 1.17. The algebra $K[x, y, z]$ possesses wild coordinates. If $\varphi=(f, g, z)$ is a $z$-wild automorphism of $K[x, y, z]$, then its nontrivial coordinates $f, g$ are both wild.

The direct application of the algorithm of Shestakov and Umirbaev from Theorem 1.14 works successfully for $z$-automorphisms. It is not clear how it may give explicit wild automorphisms which do not fix z. Theorem 1.17 of Umirbaev and $\mathrm{Yu}$ gives new examples of wild automorphisms.

Example 1.18. Let $\nu$ be the Nagata automorphism and let $\tau_{x, z}=$ $(z, y, x)$. The automorphism $\varphi=\nu \tau_{x, z} \nu$ does not fix any of the variables 
$x, y, z$. Nevertheless it is wild because $\varphi(z)=\nu(x)$ and $\nu(x)$ is a wild coordinate.

It would be interesting to give examples of wild automorphisms of $K[x, y, z]$ which cannot be obtained from Theorems 1.16, 1.17, and 1.12, applying Algorithm 1.13. There are several candidates for such wild automorphisms. All they come from the construction of Freudenburg [F1, F2 of locally nilpotent derivations which do not annulate any coordinate of $K[x, y, z]$. Among them are derivations which annulate the form $y^{2}+x z$. The simplest derivation of this kind is defined as the determinant of a Jacobian matrix

$$
\Delta(u)=\left|\begin{array}{lll}
f_{x} & g_{x} & u_{x} \\
f_{y} & g_{y} & u_{y} \\
f_{z} & g_{z} & u_{z}
\end{array}\right|,
$$

where the polynomials $f$ and $g$ are given by

$$
f=y^{2}+x z, \quad g=z f^{2}+2 x^{2} y f-x^{5} .
$$

Since $\Delta$ is locally nilpotent, $\exp (\Delta)$ is an automorphism. It does not fix any coordinate, so Theorems 1.16 and 1.17 cannot be applied directly.

Problem 1.19. Is the automorphism $\exp (\Delta)$ defined above a wild automorphism of $K[x, y, z]$ ?

We want to mention, that for $n>3$ no wild automorphisms of $K\left[x_{1}, \ldots, x_{n}\right]$ are known.

The isomorphism Aut $K[x, y] \cong A *_{C} B$ means that a set of defining relations of Aut $K[x, y]$ as an abstract group consists of the defining relations of $A$ and $B$, together with the relations which glue together the copies in $A$ and $B$ of the elements of $C$. This expresses the defining relations of Aut $K[x, y]$ in terms of the defining relations of the groups of the affine and the triangular automorphisms. The picture is much more complicated in the case of tame automorphisms of $K[x, y, z]$. Analyzing the algorithm of Theorem 1.14, very recently Umirbaev [U2] obtained a set of defining relations of TAut $K[x, y, z]$.

We write $x_{1}, x_{2}, x_{3}$ for the variables $x, y, z$ and define the automorphisms

$$
\sigma(i, \alpha, f)=\left(x_{1}, \ldots, \alpha x_{i}+f, \ldots, x_{3}\right)
$$

of the polynomial algebra $K\left[x_{1}, x_{2}, x_{3}\right]$, where $i=1,2,3, \alpha \in K^{*}$, and $f \in K\left[x_{1}, x_{2}, x_{3}\right]$ does not depend on the variable $x_{i}$. Clearly, the 
automorphisms $\sigma(i, \alpha, f)$ generate TAut $K\left[x_{1}, x_{2}, x_{3}\right]$. Let us denote

$$
\tau_{(k s)}=\sigma\left(s,-1, x_{k}\right) \sigma\left(k, 1,-x_{s}\right) \sigma\left(s, 1, x_{k}\right), \quad k, s=1,2,3, \quad k \neq s .
$$

The only nontrivial action of $\tau_{(k s)}$ is to change the variables $x_{k}$ and $x_{s}$. The defining relations of TAut $K\left[x_{1}, x_{2}, x_{3}\right]$ are given by the following important theorem.

Theorem 1.20. (Umirbaev [U2]) Let $K$ be a field of characteristic 0 . The group of tame automorphisms of $K\left[x_{1}, x_{2}, x_{3}\right]$ has the following set of defining relations with respect to the set of generators (3):

$$
\sigma(i, \alpha, f) \sigma(i, \beta, g)=\sigma(i, \alpha \beta, \beta f+g) \text {; }
$$

If $i \neq j$ and $f$ does not depend on $x_{i}, x_{j}$, then

$$
\sigma(i, \alpha, f)^{-1} \sigma(j, \beta, g) \sigma(i, \alpha, f)=\sigma\left(j, \beta, \sigma(i, \alpha, f)^{-1}(g)\right) \text {; }
$$

If $x_{j}=\tau(k s)\left(x_{i}\right)$, then

$$
\tau_{(k s)} \sigma(i, \alpha, f) \tau_{(k s)}=\sigma\left(j, \alpha, \tau_{(k s)}(f)\right) .
$$

\section{A SURVEY ON AUTOMORPHISMS OF FREE ALGEBRAS}

Let $K\left\langle x_{1}, \ldots, x_{n}\right\rangle$ be the free associative algebra freely generated by $x_{1}, \ldots, x_{n}$ over an arbitrary field $K$. We may think of it as the algebra of polynomials in $n$ noncommuting variables. As in the commutative case, the group TAut $K\left\langle x_{1}, \ldots, x_{n}\right\rangle$ of the tame automorphisms is generated by the affine and the triangular automorphisms. We start the section with the description of the automorphisms and coordinates of $K\langle x, y\rangle$. Recall that in noncommutative algebra coordinates are also called primitive elements.

Theorem 2.1. (Czerniakiewicz [Cz and Makar-Limanov [ML1, [ML2]) The automorphisms of $K\langle x, y\rangle$ are tame. The groups Aut $K\langle x, y\rangle$ and Aut $K[x, y]$ are isomorphic.

The idea of the proof is the following. The natural homomorphism

$$
\pi: K\langle x, y\rangle \rightarrow K[x, y]
$$

induces a group homomorphism

$$
\pi_{1}: \text { Aut } K\langle x, y\rangle \rightarrow \text { Aut } K[x, y]
$$

Every automorphism of $K[x, y]$ is tame and hence can be lifted to a tame automorphism of $K\langle x, y\rangle$. Hence the mapping $\pi_{1}$ is onto. It is sufficiently to show that Ker $\pi_{1}=1$. The kernel of $\pi$ is the commutator ideal of $K\langle x, y\rangle$, generated as an ideal by the commutator 
$[x, y]=x y-y x$. Hence one has to show that there are no automorphisms of $K\langle x, y\rangle$ of the form $\varphi=(x+u, y+v)$, where $u, v$ are in the commutator ideal and at least one of them is different from 0 . The proof is based on combinatorial analysis of the words in the free algebra and uses essentially the weak Euclidean algorithm. For details we refer to Chapter 6 of the book by Cohn $\mathrm{C} 2$.

The following theorem provides a simple test whether an endomorphism of $K\langle x, y\rangle$ is an automorphism.

Theorem 2.2. (Dicks Di]) The endomorphism $\varphi$ of $K\langle x, y\rangle$ is an automorphism if and only if there exists a constant $\alpha \in K^{*}$ such that

$$
[\varphi(x), \varphi(y)]=\alpha[x, y]
$$

Unfortunately the result does not give any decomposition of the automorphism as a product of affine and triangular automorphisms.

Algorithms 1.2 and 1.3 can be easily modified to recognize the automorphisms and the coordinates of $K\langle x, y\rangle$. The only difference is that one has to work in noncommutative setup. For example, in the first algorithm we have to determine whether for two homogeneous polynomials $u, v \in K\langle x, y\rangle$ one of them is a power of the other, e.g. $u=\beta v^{d}$ for some $\beta \in K^{*}$. Since $d$ is determined by the degrees of $u$ and $v$, it is sufficient to write both $u$ and $v^{d}$ as elements in $K\langle x, y\rangle$ and to see whether the corresponding monomials are proportional. In the second algorithm one has to check whether some $(d, 1)$-homogeneous polynomial is of the form $\eta\left(x+\xi y^{d}\right)$ and this also can be done easily in $K\langle x, y\rangle$.

An easier way to handle problems for the automorphisms and coordinates of $K\langle x, y\rangle$ is to reduce the considerations to similar problems in $K[x, y]$ and then to use the isomorphism (8) of the automorphism groups of $K[x, y]$ and $K\langle x, y\rangle$. This approach was applied by Shpilrain and $\mathrm{Yu}[\mathrm{SY} 2$. In particular, they found the first algorithm which recognizes the coordinates of $K\langle x, y\rangle$.

Algorithm 2.3. Let $\varphi=(f, g)$ be an endomorphism of the free algebra $K\langle x, y\rangle$.

Step 1. Consider the endomorphism $\psi=(\pi(f), \pi(g))$ of $K[x, y]$, where $\pi(f)$ and $\pi(g)$ are the abelianization of $f$ and $g$ from (17). Apply Algorithm 1.2 to $\psi$. If $\psi$ is not an automorphism of $K[x, y]$, then $\varphi$ is not an automorphism of $K\langle x, y\rangle$. 
Step 2. If $\psi$ is an automorphism, decompose it as a product $\psi=$ $\psi_{1} \cdots \psi_{n}$ of affine and triangular automorphisms $\psi_{i}$. Consider the automorphism

$$
\pi_{1}^{-1}(\psi)=\pi_{1}^{-1}\left(\psi_{1}\right) \cdots \pi_{1}^{-1}\left(\psi_{n}\right) .
$$

Then $\varphi$ is an automorphism if and only if $\varphi=\pi_{1}^{-1}(\psi)$.

Algorithm 2.4. (Shpilrain and Yu SY2]) Let $f \in K\langle x, y\rangle$.

Step 1. Consider the abelianization $\pi(f)$ of $f$. Apply Algorithm 1.3. or 1.4 if char $K=0$, to decide whether $\pi(f)$ is a coordinate of $K[x, y]$. If it is not, then $f$ is not a coordinate of $K\langle x, y\rangle$.

Step 2. If $\pi(f)$ is a coordinate of $K[x, y]$, find an automorphism $\psi$ of $K[x, y]$ which sends $x$ to $\pi(f)$. Then $f$ is a coordinate if and only if $\varphi=\pi_{1}^{-1}(\psi)$ sends $x$ to $f$.

For the motivation of the algorithm, let $\varphi=\pi_{1}^{-1}(\psi) \in$ Aut $K\langle x, y\rangle$, where $\psi \in$ Aut $K[x, y]$ sends $x$ to $\pi(f)$. All automorphisms $\psi^{\prime} \in$ Aut $K[x, y]$ with this property are of the form $\psi^{\prime}=\psi \cdot(x, \beta y+h(x))$, $\beta \in K^{*}, h(x) \in K[x]$. Hence

$$
\varphi^{\prime}=\pi_{1}^{-1}\left(\psi^{\prime}\right)=\pi_{1}^{-1}(\psi) \cdot(x, \beta y+h(x)) \varphi \cdot(x, \beta y+h(x))
$$

and $\varphi(x)=\varphi^{\prime}(x)$. See [SY2] also for other properties of the coordinates of $K\langle x, y\rangle$.

As in the commutative case, see Wi, [MLSY], one considers the problem whether two polynomials $p, q \in K\langle x, y\rangle$ are automorphically equivalent. The corresponding algorithm is given by Drensky and Yu DY6].

By analogy with the commutative case one can ask immediately the following problem. It is stated, for example, by Cohn as Problem 1.74 in $[\mathrm{DN}]$.

Problem 2.5. Is every automorphism of $K\left\langle x_{1}, \ldots, x_{n}\right\rangle, n>2$, tame?

If we are able to lift any wild automorphism of $K[x, y, z]$ to an automorphism of $K\langle x, y, z\rangle$, then we shall obtain automatically a wild automorphism. Up till now, no such wild automorphism has been constructed.

Problem 2.6. Can the wild automorphisms of $K[x, y, z]$ be lifted to automorphisms of $K\langle x, y, z\rangle$ ? Can the Nagata automorphism be lifted? Can the wild z-automorphisms of $K[z][x, y]$ be lifted to z-automorphisms of $K\langle x, y, z\rangle$ (or at least to any automorphisms of $K\langle x, y, z\rangle$, not necessarily fixing $z)$ ? 
A stronger version of this problem is the following.

Problem 2.7. Can every wild coordinate of $K[x, y, z]$ be lifted to a coordinate of $K\langle x, y, z\rangle$ ? Can the two nontrivial coordinates of the Nagata automorphism be lifted? Can the two nontrivial coordinates of every wild z-automorphism of $K[z][x, y]$ be lifted to coordinates of a $z$-automorphism of $K\langle x, y, z\rangle$ ?

The most prospective candidate for a wild automorphism of the free algebra with three generators is the example of Anick $(x+(y) x y-$ $y z), y, z+(z y-y z) y)$, see the book by Cohn [C2], p. 343, or, $(x+$ $z(x z-z y), y+(x z-z y) z, z)$, changing the places of $y$ and $z$.

Conjecture 2.8. The Anick automorphism

$$
\nu_{1}=(x+z(x z-z y), y+(x z-z y) z, z)
$$

of $K\langle x, y, z\rangle$ is wild.

The Anick automorphism fixes $z$. Hence we may consider the variable $z$ as a noncommutative constant and treat the Anick automorphism as a $z$-automorphism of $K\langle x, y, z\rangle$. It has the property that the first two coordinates are linear in $x$ and $y$. We call such automorphisms linear $z$-automorphisms. From some point of view, the linear $z$-automorphisms are the simplest $z$-automorphisms. It is natural to expect the appearance of some group of matrices there.

There is a general concept of partial derivatives and the Jacobian matrix in the free algebra, developed by Yagzhev $[\mathrm{Y}]$, Dicks and Lewin DiL], and Schofield [Sc], including the solution of the Jacobian conjecture for free associative algebras for $n=2$ in [DiL] and arbitrary $n$ in Sc.

For the study of linear z-automorphisms, Drensky and Yu DY3 introduced a simplified version of the partial derivatives of Dicks and Lewin DiL. We shall restrict our considerations to the free algebra with three generators only. Let $f \in K\langle x, y, z\rangle$ be linear in $x, y$. Then it has the form

$$
f=\sum \alpha_{i j} z^{i} x z^{j}+\sum \beta_{i j} z^{i} y z^{j}, \quad \alpha_{i j}, \beta_{i j} \in K .
$$

The $z$-derivatives $f_{x}$ and $f_{y}$ are defined by

$$
f_{x}=\sum \alpha_{i j} z_{1}^{i} z_{2}^{j}, \quad f_{y}=\sum \beta_{i j} z_{1}^{i} z_{2}^{j}
$$

Here $f_{x}$ and $f_{y}$ are in $K\left[z_{1}, z_{2}\right]$ and are polynomials in two commuting variables. The $z$-Jacobian matrix of the linear $z$-endomorphism $\varphi=$ 
$(f, g)$ of $K\langle x, y, z\rangle$ is defined as

$$
J_{z}(\varphi)=\left(\begin{array}{ll}
f_{x} & g_{x} \\
f_{y} & g_{y}
\end{array}\right) .
$$

It is easy to see that the linear $z$-endomorphism $\varphi$ of $K\langle x, y, z\rangle$ is an automorphism if and only if the matrix $J_{z}(\varphi)$ is invertible as a matrix with entries in $K\left[z_{1}, z_{2}\right]$. Also, the group of linear $z$-automorphisms is isomorphic to $G L_{2}\left(K\left[z_{1}, z_{2}\right]\right)$. The following theorem was established in DY3.

Theorem 2.9. (i) A linear z-automorphism is z-tame if and only if its z-Jacobian matrix belongs to $G E_{2}\left(K\left[z_{1}, z_{2}\right]\right)$.

(ii) Every linear $z$-automorphism of $K\langle x, y, z\rangle$ induces a tame automorphism of $K[x, y, z]$.

Applying the result of Tolhuizen, Hollmann and Kalker [THK] and Park $[\mathrm{P}$ we can decide whether a linear $z$-automorphism is $z$-tame applying the Euclidean division algorithm to the $z$-Jacobian matrix. We apply this to the Anick automorphism.

Corollary 2.10. The Anick automorphism is z-wild.

Really, the $z$-Jacobian matrix of the Anick automorphism is

$$
J_{z}\left(\nu_{1}\right)=\left(\begin{array}{cc}
1+z_{1} z_{2} & z_{2}^{2} \\
-z_{1}^{2} & 1-z_{1} z_{2}
\end{array}\right) .
$$

The matrix $J_{z}\left(\nu_{1}\right)$ coincides with the well known example of Cohn C1] of a matrix in $G L_{2}\left(K\left[z_{1}, z_{2}\right]\right)$ which does not belong to $G E_{2}\left(K\left[z_{1}, z_{2}\right]\right)$. (Direct arguments: Since the homogeneous components of second (maximal) degree of the first column of $J_{z}\left(\nu_{1}\right)$ do not divide each other, we cannot apply the Euclidean algorithm.) Hence the Anick automorphism is $z$-wild. The same arguments work for the matrix

$$
J_{z}(\varphi)=\left(\begin{array}{cc}
1+z_{1} z_{2} h\left(z_{1}, z_{2}\right) & z_{2}^{2} h\left(z_{1}, z_{2}\right) \\
-z_{1}^{2} h\left(z_{1}, z_{2}\right) & 1-z_{1} z_{2} h\left(z_{1}, z_{2}\right)
\end{array}\right)
$$

where $h\left(z_{1}, z_{2}\right)$ is any nonzero polynomial in $K\left[z_{1}, z_{2}\right]$. Then the corresponding automorphism $\varphi$ is $z$-wild.

By the celebrated theorem of Suslin $\left[\mathbf{S u}\right.$, every matrix in $G L_{n}\left(K\left[z_{1}, \ldots, z_{p}\right]\right)$, $n \geq 3$, can be presented as a product of a diagonal matrix and elementary matrices. Hence, for $n \geq 3$ all linear $z$-automorphisms of $K\left\langle x_{1}, \ldots, x_{n}, z\right\rangle$ are tame. In particular, the linear $z$-automorphisms of $K\langle x, y, z\rangle$ are stably tame. For example, from a lemma of Mennicke, used in the constructive proof of the Suslin theorem given by 
Park and Woodburn PW], follows that, extended by $\nu_{1}(t)=t$, the Anick automorphism of $K\langle x, y, t, z\rangle$ can be decomposed as a product of "elementary" $z$-automorphisms

$$
\nu_{1}=\varepsilon_{31}\left(z_{1}\right) \varepsilon_{32}\left(z_{2}\right) \varepsilon_{13}\left(-z_{2}\right) \varepsilon_{23}\left(z_{1}\right) \varepsilon_{31}\left(-z_{1}\right) \varepsilon_{32}\left(-z_{2}\right) \varepsilon_{13}\left(z_{2}\right) \varepsilon_{23}\left(-z_{1}\right),
$$

where the the $z$-automorphism $\varepsilon_{i j}\left(\alpha z_{1}^{a} z_{2}^{b}\right)$ of $K\langle x, y, t, z\rangle$ is defined by

$$
\varepsilon_{i j}\left(\alpha z_{1}^{a} z_{2}^{b}\right): x_{j} \rightarrow x_{j}+\alpha z^{a} x_{i} z^{b}, \quad \varepsilon_{i j}\left(\alpha z_{1}^{a} z_{2}^{b}\right): x_{k} \rightarrow x_{k}, \quad k \neq j,
$$

and $x_{1}=x, x_{2}=y, x_{3}=t$.

The proof of Theorem 2.9] in DY3 gives a criterion for $z$-wildness of a class of nonlinear $z$-automorphisms. See the discussions below on our results in [DY4, DY5] for further development of the idea.

Corollary 2.11. Let $\varphi=(f, g)$ be a z-automorphism of $K\langle x, y, z\rangle$. Let $\varphi_{1}=\left(f_{1}, g_{1}\right)$, where $f_{1}, g_{1}$ are the linear in $x$ and $y$ components of $f, g$. If the matrix $J_{z}\left(\varphi_{1}\right)$ does not belong to $G E_{2}\left(K\left[z_{1}, z_{2}\right]\right)$, then the automorphism $\varphi$ is z-wild.

The study of $z$-tame automorphisms of $K\langle x, y, z\rangle$ has continued in the very recent paper by Drensky and Yu DY7. The following result is an analogue of Theorem 1.10 (i) established by Wright $[\mathrm{Wr}$. It shows that the structure of the group of $z$-tame automorphisms of $K\langle x, y, z\rangle$ is similar to the structure of the group of $z$-tame automorphisms of $K[x, y, z]$.

Theorem 2.12. (Drensky and Yu DY7]) Over an arbitrary field $K$, the group TAut $_{z} K\langle x, y, z\rangle$ of $z$-tame automorphisms of $K\langle x, y, z\rangle$ is isomorphic to the free product $A *_{C} B$ of the group $A$ of the $z$-affine automorphisms and the group $B$ of $z$-triangular automorphisms amalgamating their intersection $C=A \cap B$.

Since the group of the $z$-automorphisms which are linear in $x, y$ is isomorphic to the group $G L_{2}\left(K\left[z_{1}, z_{2}\right]\right)$, we immediately obtain:

Corollary 2.13. The group TAut $_{z} K\langle x, y, z\rangle$ is isomorphic to the free product with amalgamation $G E_{2}\left(K\left[z_{1}, z_{2}\right]\right) *_{C_{1}} B$, where $G E_{2}\left(K\left[z_{1}, z_{2}\right]\right)$ is identified as above with the group of $z$-tame automorphisms which are linear in $x$ and $y, B$ is the group of $z$-triangular automorphisms and $C_{1}=G E_{2}\left(K\left[z_{1}, z_{2}\right]\right) \cap B$.

Now we can use Theorem 2.12 to present algorithms which recognize $z$-tame automorphisms and coordinates of $K\langle x, y, z\rangle$. We start with an algorithm which determines whether a $z$-endomorphism of $K\langle x, y, z\rangle$ is 
a $z$-tame automorphism. The main idea is similar to that of Algorithm 1.2 which decides whether an endomorphism of $K[x, y]$ is an automorphism, but the realization is more sophisticated. Instead of the usual degree used there we define a bidegree of $K\langle x, y, z\rangle$ assuming that the monomial $w$ is of bidegree bideg $w=(d, e)$ if $\operatorname{deg}_{x} w+\operatorname{deg}_{y} w=d$ and $\operatorname{deg}_{z} w=e$. We order the bidegrees $(d, e)$ lexicographically, i.e., $\left(d_{1}, e_{1}\right)>\left(d_{2}, e_{2}\right)$ means that either $d_{1}>d_{2}$ or $d_{1}=d_{2}$ and $e_{1}>e_{2}$. In order to simplify further the considerations, we use the trick introduced by Formanek $[\mathrm{Fo}$ in his construction of central polynomials of matrices.

Let $H_{n}$ be the subspace of $K\langle x, y, z\rangle$ consisting of all polynomials which are homogeneous of degree $n$ with respect to $x$ and $y$. We define an action of $K\left[t_{0}, t_{1}, \ldots, t_{n}\right]$ on $H_{n}$ in the following way. If

$$
w=z^{a_{0}} u_{1} z^{a_{1}} u_{2} \cdots z^{a_{n-1}} u_{n} z^{a_{n}},
$$

where $u_{i}=x$ or $u_{i}=y, i=1, \ldots, n$, then

$$
t_{0}^{b_{0}} t_{1}^{b_{1}} \cdots t_{n}^{b_{n}} * w=z^{a_{0}+b_{0}} u_{1} z^{a_{1}+b_{1}} u_{2} \cdots z^{a_{n-1}+b_{n-1}} u_{n} z^{a_{n}+b_{n}},
$$

and extend this action by linearity. Clearly, $H_{n}$ is a free $K\left[t_{0}, t_{1}, \ldots, t_{n}\right]$ module with basis consisting of the $2^{n}$ monomials $u_{1} \cdots u_{n}$, where $u_{i}=$ $x$ or $u_{i}=y$. The proofs of the following equations are obtained by easy direct computation. Let $\beta \in K^{*}$,

$$
\begin{gathered}
v(x, y, z)=\sum \theta_{i}\left(t_{0}, t_{1}, \ldots, t_{k}\right) * u_{i_{1}} \cdots u_{i_{k}} \in H_{k}, \\
q(y, z)=\omega\left(t_{0}, t_{1}, \ldots, t_{d}\right) * y^{d} \in H_{d},
\end{gathered}
$$

where $\theta_{i} \in K\left[t_{0}, t_{1}, \ldots, t_{k}\right], \omega \in K\left[t_{0}, t_{1}, \ldots, t_{d}\right], u_{i_{j}}=x$ or $u_{i_{j}}=y$. Then

$$
\begin{gathered}
u(x, y, z)=q(v(x, y, z) / \beta, z)=\omega\left(t_{0}, t_{d}, t_{2 d}, \ldots, t_{k d}\right) / \beta^{d} \\
\left(\sum \theta_{i}\left(t_{0}, t_{1}, \ldots, t_{k}\right) * u_{i_{1}} \cdots u_{i_{k}}\right) \\
\left(\sum \theta_{i}\left(t_{k}, t_{k+1}, \ldots, t_{2 k}\right) * u_{i_{1}} \cdots u_{i_{k}}\right) \cdots \\
\left(\sum \theta_{i}\left(t_{k(d-1)}, t_{k(d-1)+1}, \ldots, t_{k d}\right) * u_{i_{1}} \cdots u_{i_{k}}\right) .
\end{gathered}
$$

Algorithm 2.14. (Drensky and Yu [DY7]) Let $\varphi=(f, g)$ be a $z$ endomorphism of $K\langle x, y, z\rangle$. We make use of the bidegree defined above. 
Step 0. If some of the polynomials $f, g$ depends on $z$ only, then $\varphi$ is not an automorphism.

Step 1. Let $u, v$ be the homogeneous components of highest bidegree of $f, g$, respectively. If both $u, v$ are of bidegree $(1,0)$, i.e., linear, then we check whether they are linearly independent. If yes, then $\varphi$ is a product of a linear automorphism (from $G L_{2}(K)$ ) and a translation $(x+p(z), y+r(z))$. If $u, v$ are linearly dependent, then $\varphi$ is not an automorphism.

Step 2. Let bideg $u>(1,0)$ and bideg $u \geq \operatorname{bideg} v$. Hence $u \in H_{l}$, $v \in H_{k}$ for some $k$ and $l$. We have to check whether $l=k d$ for a positive integer $d$ and to decide whether $u=q(v / \beta, z)$ for some $\beta \in K^{*}$ and some $q(y, z) \in H_{d}$. We know $u$ in (14) and $v$ in (12) up to the multiplicative constant $\beta$. Hence, up to $\beta$, we know the polynomials $\theta_{i}\left(t_{0}, t_{1}, \ldots, t_{n}\right)$ in the presentation of $v$. We compare some of the nonzero polynomial coefficients of $u=\sum \lambda_{j}\left(t_{0}, \ldots, t_{k d}\right) u_{j_{1}} \cdots u_{i_{k d}}$ with the corresponding coefficient of $q(v / \beta, z)$. We can find explicitly, up to the value of $\beta^{d}$, the polynomial $\omega\left(t_{0}, t_{1}, \ldots, \omega_{d}\right)$ in (13) using the usual division of polynomials. If $l=k d$ and $u=q(v / \beta, z)$, then we replace $\varphi=(f, g)$ with $\varphi_{1}=(f-q(g / \beta, z), g)$. Then we apply Step 0 to $\varphi_{1}$. If $u$ cannot be presented in the desired form, then $\varphi$ is not an automorphism.

Step 3. If bideg $v>(1,0)$ and bideg $u<\operatorname{bideg} v$, we have similar considerations, as in Step 2, replacing $\varphi=(f, g)$ with $\varphi_{1}=(f, g-$ $q(f / \alpha, z))$ for a suitable $q(y, z)$. Then we apply Step 0 to $\varphi_{1}$. If $v$ cannot be presented in this form, then $\varphi$ is not an automorphism.

The following example from [DY4, DY5, DY7] is based on the polynomial $x z-z y$ which appears in the Anick automorphism.

Example 2.15. Let $h(t, z) \in K\langle t, z\rangle$. Then

$$
\sigma_{h}=(x+z h(x z-z y, z), y+h(x z-z y, z) z, z)
$$

is an automorphism of $K\langle x, y, z\rangle$ fixing $x z-z y$. Its inverse is $\sigma_{-h}$.

Corollary 2.16. (Drensky and Yu [DY7]) Let $h(t, z) \in K\langle t, z\rangle$ and let $\operatorname{deg}_{u} h(u, z)>0$. Then

$$
\sigma_{h}=(x+z h(x z-z y, z), y+h(x z-z y, z) z, z)
$$

is a z-wild automorphism of $K\langle x, y, z\rangle$.

For the proof, we apply Algorithm 2.14. Let $w$ be the homogeneous component of highest bidegree of $h(x z-z y, z)$. Clearly, $w$ has the form 
$w=\bar{h}(x z-z y, z)=q(x z-z y, z)$ for some bihomogeneous polynomial $q(t, z) \in K\langle t, z\rangle$. The leading components of the coordinates of $\sigma_{h}$ are $z q(x z-z y, z)$ and $q(x z-z y, z) z$, and are of the same bidegree. If $\sigma_{h}$ is a $z$-tame automorphism, then we can reduce the bidegree using a linear transformation, which is impossible because $z q(x z-z y, z)$ and $q(x z-z y, z) z$ are linearly independent.

Clearly, if $\varphi=(f, g)$ is a $z$-automorphism of $K\langle x, y, z\rangle$, with the same first coordinate, then we can find all other $z$-coordinate mates of $f$. This argument and Corollary 2.16 give immediately:

Corollary 2.17. (Drensky and Yu [DY7]) Let $h(t, z) \in K\langle t, z\rangle$ and let $\operatorname{deg}_{u} h(u, z)>0$. Then $f(x, y, z)=x+z h(x z-z y, z)$ is a z-wild coordinate of $K\langle x, y, z\rangle$.

Now we are able to modify Algorithm 2.14 to decide whether a polynomial $f(x, y, z)$ is a $z$-tame coordinate of $K\langle x, y, z\rangle$. We refer to DY7] for details.

Theorem 2.18. (Drensky and Yu [DY7]) There is an algorithm which decides whether a polynomial $f(x, y, z) \in K\langle x, y, z\rangle$ is a $z$-tame coordinate.

As we already mentioned in the comments after Theorem [2.1] the main step of the proof of Czerniakiewicz $[\mathrm{Cz}$ and Makar-Limanov ML1, ML2 for the tameness of the automorphisms of $K\langle x, y\rangle$ is the following. If $\varphi=(x+u, y+v)$ is an endomorphism of $K\langle x, y\rangle$, where $u, v$ are in the commutator ideal of $K\langle x, y\rangle$ and at least one of them is different from 0 , then $\varphi$ is not an automorphism of $K\langle x, y\rangle$. The condition that $u(x, y)$ and $v(x, y)$ belong to the commutator ideal of $K\langle x, y\rangle$ immediately implies that all monomials of $u$ and $v$ depend on both $x$ and $y$, as required in the following theorem.

Theorem 2.19. (Drensky and Yu DY7]) The z-endomorphisms of the form

$$
\varphi=(x+u(x, y, z), y+v(x, y, z)),
$$

where $(u, v) \neq(0,0)$ and all monomials of $u$ and $v$ depend on both $x$ and $y$, are not automorphisms of $K\langle x, y, z\rangle$.

Till the end of the section we fix the field $K$ of characteristic 0 and continue our survey with the work of Umirbaev [U2] devoted on the wildness of the Anick automorphism. It is too difficult to controll the behavior of the tame automorphisms of $K\langle x, y, z\rangle$. Instead, it is more 
convenient to consider some factor algebra of $K\langle x, y, z\rangle$ modulo an ideal of $K\langle x, y, z\rangle$ which is invariant under the action of the automorphism group of $K\langle x, y, z\rangle$. This ideal should be big enough to allow to work in the factor algebra, and not too big, in order to preserve the wildness of some automorphisms. The ideals of the free algebra which are invariant under all endomorphisms are called T-ideals. Such an ideal $U$ of $K\left\langle x_{1}, \ldots, x_{n}\right\rangle$ can be characterized with the property that there exists an associative algebra $R$ such that $U$ coincides with the ideal of all polynomial polynomial identities $u\left(x_{1}, \ldots, x_{n}\right)=0$ of $R$. This means that $u\left(r_{1}, \ldots, r_{n}\right)=0$ for all $r_{1}, \ldots, r_{n} \in R$. The factor algebra $K\left\langle x_{1}, \ldots, x_{n}\right\rangle / U$ is called the (relatively) free algebra of rank $n$ in the variety of algebras generated by the algebra $R$, or defined by the polynomial identities from $U$.

The obtained results have shown that it is very convenient to work in the free metabelian associative algebra which we denote by $C_{n}$. The variety of metabelian associative algebras is determined by the polynomial identity $\left[t_{1}, t_{2}\right]\left[t_{3}, t_{4}\right]=0$. One can define partial derivatives and the related Jacobian matrix induced by the corresponding objects in the free associative algebra. Although $C_{n}$ is a homomorphic image of $K\left\langle x_{1}, \ldots, x_{n}\right\rangle$, we denote its generators with the same symbols $x_{1}, \ldots, x_{n}$. For the partial derivatives we need two sets of commuting variables $\left\{u_{1}, \ldots, u_{n}\right\}$ and $\left\{v_{1}, \ldots, v_{n}\right\}$. We define formal partial derivatives $\partial_{M} / \partial_{M} x_{i}$ by

$$
\frac{\partial_{M} x_{i}}{\partial_{M} x_{i}}=1, \quad \frac{\partial_{M} x_{j}}{\partial_{M} x_{i}}=0, j \neq i,
$$

and, for a monomial $w=x_{i_{1}} \cdots x_{i_{m}} \in C_{n}$,

$$
\frac{\partial_{M} w}{\partial_{M} x_{i}}=\sum_{k=1}^{m} u_{i_{1}} \cdots u_{i_{k-1}} v_{i_{k+1}} \ldots v_{i_{m}} \frac{\partial_{M} x_{i_{k}}}{\partial_{M} x_{i}} .
$$

A polynomial $f \in C_{n}$ belongs to the commutator ideal of $C_{n}$, i.e., to the kernel of the natural homomorphism $C_{n} \rightarrow K\left[x_{1}, \ldots, x_{n}\right]$, if and only if

$$
\sum_{i=1}^{n}\left(u_{i}-v_{i}\right) \frac{\partial_{M} f}{\partial_{M} x_{i}}=0 .
$$

The Jacobian matrix of an endomorphism $\varphi$ of $C_{n}$ is

$$
J_{M}(\varphi)=\left(\frac{\partial_{M} \varphi\left(x_{j}\right)}{\partial_{M} x_{i}}\right),
$$


which is a matrix with entries from the polynomial algebra $K\left[u_{1}, \ldots, u_{n}, v_{1}, \ldots, v_{n}\right]$. Umirbaev U1 proved that the Jacobian matrix $J_{M}(\varphi)$ is invertible (as a matrix with polynomial entries) if and only if $\varphi$ is an automorphism of $C_{n}$. Also, every automorphism of $K\left[x_{1}, \ldots, x_{n}\right]$ can be lifted to an automorphism of $C_{n}$. Clearly, the invertibility of $J_{M}(\varphi)$ is equivalent to $0 \neq \operatorname{det}\left(J_{M}(\varphi)\right) \in K$.

We shall work with free algebras of rank 3 only and shall use the sets of variables

$$
X=\{x, y, z\}, \quad U=\left\{x_{1}, y_{1}, z_{1}\right\}, \quad V=\left\{x_{2}, y_{2}, z_{2}\right\} .
$$

instead of $X=\left\{x_{1}, x_{2}, x_{3}\right\}, U=\left\{u_{1}, u_{2}, u_{3}\right\}, V=\left\{v_{1}, v_{2}, v_{3}\right\}$, respectively, and shall denote the algebra $C_{3}$ by $C$. For example, if $f \in C$ is linear in $x$ and $y$, it can be written in the form (91) and its partial derivatives $\partial_{M} f / \partial_{M} x$ and $\partial_{M} f / \partial_{M} y$ coincide, respectively, with $f_{x}$ and $f_{y}$ in (10). Hence, if $\varphi=(f, g, z)$ is a $z$-endomorphism of $C$, and $f, g$ are linear in $x, y$, then the Jacobian matrix $J_{M}(\varphi)$ is of the form

$$
J_{M}(\varphi)=\left(\begin{array}{ccc}
f_{x} & g_{x} & 0 \\
f_{y} & g_{y} & 0 \\
\partial_{M} f / \partial_{M} z & \partial_{M} g / \partial_{M} z & 1
\end{array}\right)
$$

compare it with $J_{z}(\varphi)$ from (11).

There is a natural homomorphism

$$
\text { TAut } K\langle x, y, z\rangle \rightarrow \operatorname{TAut}(C) \rightarrow \text { TAut } K[x, y, z] .
$$

As a consequence of Theorem 1.20, Umirbaev [U2] obtained the following.

Proposition 2.20. As a normal subgroup of TAut $(C)$, the kernel of the homomorphism

$$
\operatorname{TAut}(C) \rightarrow \text { TAut } K[x, y, z]
$$

is generated by the automorphisms

$\psi_{w}=(x+w(y, z), y, z), \quad w(y, z)=\sum_{p, q, r, s \geq 0} \alpha_{p q r s} y^{p} z^{q}[y, z] y^{r} z^{s}, \alpha_{p q r s} \in K$.

Hence the kernel of the homomorphism TAut $(C) \rightarrow$ TAut $K[x, y, z]$ is generated by automorphisms of the form

$$
\vartheta=\sigma\left(i_{k}, \alpha_{k}, g_{k}\right)^{-1} \cdots \sigma\left(i_{1}, \alpha_{1}, g_{1}\right)^{-1} \psi_{w} \sigma\left(i_{1}, \alpha_{1}, g_{1}\right) \cdots \sigma\left(i_{k}, \alpha_{k}, g_{k}\right)
$$


for some automorphisms $\sigma\left(i_{j}, \alpha_{j}, g_{j}\right)$ from (3). The Jacobian matrix of $\psi_{f}$ is of the form

$$
J_{M}\left(\psi_{w}\right)=\left(\begin{array}{ccc}
1 & 0 & 0 \\
\partial_{M} w / \partial_{M} y & 1 & 0 \\
\partial_{M} w / \partial_{M} z & 0 & 1
\end{array}\right),
$$

which $\sigma\left(i_{j}, \alpha_{j}, g_{j}\right)$ also has an elementary Jacobian matrix. Unfortunately, this fact cannot be applied directly to recognize the tame automorphisms due to the validity of the Suslin theorem $[\mathrm{Su}]$ for $n \geq 3$. On the other hand, in the case of the Anick automorphism and other linear $z$-automorphisms $\varphi$ the relation between $J_{z}(\varphi)$ from (11) and $J_{M}(\varphi)$ from (15) is obvious. Hence it is natural to try to apply the idea of Theorem 2.9 and to reduce the considerations to the $2 \times 2$ case. This was done by Umirbaev [U2] in the following way. For an automorphism $\varphi=(f, g, h)$ of $C$, he introduced the $2 \times 2$ "Jacobian" matrix

$$
J_{2}(\varphi)=\left(\begin{array}{ll}
\partial_{M} f / \partial_{M} x & \partial_{M} g / \partial_{M} x \\
\partial_{M} f / \partial_{M} y & \partial_{M} g / \partial_{M} y
\end{array}\right)
$$

Then he defined a homomorphism $\eta: K\left[z_{1}, y_{1}, z_{1}, x_{2}, y_{2}, z_{2}\right] \rightarrow K\left[z_{1}, z_{2}\right]$ by

$$
\eta\left(x_{1}\right)=\eta\left(y_{1}\right)=\eta\left(x_{2}\right)=\eta\left(y_{2}\right)=0, \quad \eta\left(z_{1}\right)=z_{1}, \quad \eta\left(z_{2}\right)=z_{2} .
$$

Using the concrete form of the generators (3) of TAut $K[x, y, z]$ and the defining relations (44), (피), and (6) between them, Umirbaev established that for the automorphism $\vartheta$ from (17) the matrix $\eta\left(J_{2}(\vartheta)\right)$ belongs to $G E_{2}\left(K\left[z_{1}, z_{2}\right]\right)$. This implies the following theorem.

Theorem 2.21. (Umirbaev U2]) Let the field $K$ be of characteristic 0 and let $\varphi$ be a tame automorphism of the free metabelian algebra $C$ which induces the identity automorphism of $K[x, y, z]$. Then the matrix $\eta\left(J_{2}(\varphi)\right)$ belongs to the group $G E_{2}\left(K\left[z_{1}, z_{2}\right]\right)$.

Now, there is only one obvious step left to the proof of the wildness of the Anick automorphism. Consider a tame linear $z$-automorphism $\vartheta$ of $K\langle x, y, z\rangle$ which induces the same automorphism of $K[x, y, z]$ as the Anick automorphism $\nu_{1}$. One can apply Theorem 2.21 to $\vartheta^{-1} \nu_{1}$. But the matrix

$$
\eta\left(J_{2}\left(\vartheta^{-1} \nu_{1}\right)\right)=\eta\left(J_{2}\left(\vartheta^{-1}\right)\right) \eta\left(J_{2}\left(\nu_{1}\right)\right)
$$

does not belong to $G E_{2}\left(K\left[z_{1}, z_{2}\right]\right)$. 
Theorem 2.22. (Umirbaev U2]) The Anick automorphism

$$
\nu_{1}=(x+z(x z-z y), y+(x z-z y) z, z)
$$

is a wild automorphism of the free metabelian algebra $C$ and hence of the free associative algebra $K\langle x, y, z\rangle$.

In the same way Umirbaev showed the following theorem which replaces the $z$-wildness of Theorem 2.9 with wildness in the usual sense.

Theorem 2.23. A linear $z$-automorphism of $K\langle x, y, z\rangle$ is tame as an automorphism of $K\langle x, y, z\rangle$ if and only if its $z$-Jacobian matrix belongs to $G E_{2}\left(K\left[z_{1}, z_{2}\right]\right)$.

As in the case of polynomial algebras, the next step of studying automorphisms of free algebras is to study coordinates, or primitive elements. In particular, the following problem is motivated by [UY]. We state it in the case of three variables only, see DY4, DY5 for the general case.

Problem 2.24. If $f(x, y, z) \in K\langle x, y, z\rangle$ is an image of $x$ under a $z$-wild automorphism, is there a tame automorphism (maybe not fixing z) which also sends $x$ to $f(x, y, z)$ ?

If such a tame automorphism does not exist, we call $f(x, y, z)$ a wild coordinate of $K\langle x, y, z\rangle$.

As an analog of the Strong Nagata Conjecture in [UY], we state

Conjecture 2.25. (Strong Anick Conjecture) There exist wild coordinates in $K\langle x, y, z\rangle$. In particular, the two nontrivial coordinates of the Anick automorphism are both wild.

The following theorem is one of the main results in Drensky and $\mathrm{Yu}$ DY4, DY5].

Theorem 2.26. Let $K$ be a field of characteristic 0 and let the polynomial $f(x, y, z) \in K\langle x, y, z\rangle$ be linear in $x, y$. If there exists a wild automorphism of $K\langle x, y, z\rangle$ which fixes $z$ and sends $x$ to $f(x, y, z)$, then every automorphism of $K\langle x, y, z\rangle$ which sends $x$ to $f(x, y, z)$ is also wild. So, $f(x, y, z)$ is a wild coordinate of $K\langle x, y, z\rangle$.

Theorem 2.26 gives an algorithm deciding whether a polynomial $f(x, y, z) \in K\langle x, y, z\rangle$ which is linear in $x$ and $y$, is a tame coordinate. If it is, then the algorithm finds a product of $z$-elementary automorphisms 
which sends $x$ to $f(x, y, z)$. (Of course, as in the other algorithmic considerations we assume that the ground field $K$ is constructive, and we may perform calculations there.) For this purpose, it is sufficient to combine Theorem 2.26 with the following convenient form of the result in [P, THK], as stated in THK]. Let $a\left(z_{1}, z_{2}\right), b\left(z_{1}, z_{2}\right)$ be two polynomials in $K\left[z_{1}, z_{2}\right]$. Then there exist $c\left(z_{1}, z_{2}\right), d\left(z_{1}, z_{2}\right) \in K\left[z_{1}, z_{2}\right]$ such that the matrix

$$
G=\left(\begin{array}{ll}
a & c \\
b & d
\end{array}\right)
$$

belongs to $G E_{2}\left(K\left[z_{1}, z_{2}\right]\right)$ if and only if we can bring the pair $(a, b)$ to $(\alpha, 0), \alpha \in K^{*}$, using the Euclidean algorithm only.

The following consequence of Theorem 2.26 gives the affirmative answer to the Strong Anick Conjecture.

Theorem 2.27. (Drensky and Yu [DY4, DY5]) The Strong Anick Conjecture is true. Namely, there exist wild coordinates in $K\langle x, y, z\rangle$. In particular, the two nontrivial coordinates $x+z(x z-z y)$ and $y+(x z-$ zy) z of the Anick automorphism

$$
\omega=(x+z(x z-z y), y+(x z-z y) z, z)
$$

are both wild.

We call an automorphism $\varphi=(f(x, y, z), g(x, y, z), z)$ of $K\langle x, y, z\rangle$ Anick-like if $f(x, y, z)$ and $g(x, y, z)$ are linear in $x, y$ and the matrix $J_{z}(\varphi)$ does not belong to $G E_{2}\left(K\left[z_{1}, z_{2}\right]\right)$. The following corollary from DY4, DY5] is an analogue of a result from [UY].

Corollary 2.28. The two nontrivial coordinates $f(x, y, z), g(x, y, z)$ of any Anick-like automorphism

$$
\varphi=(f(x, y, z), g(x, y, z), z)
$$

of $K\langle x, y, z\rangle$ are wild.

In the spirit of the above results, we obtain in [DY4, DY5] the following theorem which is much stronger.

Theorem 2.29. Let $f(x, y, z)$ be a z-coordinate of $K\langle x, y, z\rangle$ without terms depending only on $z$ (i.e., $f(0,0, z)=0)$. If the linear part (with respect to $x$ and $y) f_{1}(x, y, z)$ of $f(x, y, z)$ is a $z$-wild coordinate, then $f(x, y, z)$ itself is also a wild coordinate of $K\langle x, y, z\rangle$.

The restriction $f(0,0, z)=0$ is essential for the proof of Theorem 2.29. Nevertheless, it seems very unlikely to have a wild automorphism 
$(f, g, z)$ with $f(0,0, z)=0$ such that $f(x, y, z)+a(z)$ is a tame coordinate for some polynomial $a(z)$ in view of the next theorem.

Theorem 2.30. (Drensky and Yu DY4, DY5]) Let $(f, g, z)$ be an automorphism of $K\langle x, y, z\rangle$ and let the linear part (with respect to $x$ and $y$ ) of it, $\left(f_{1}, g_{1}, z\right)$, be a z-wild automorphism. Then $(f, g, z)$ is also a wild automorphism of $K\langle x, y, z\rangle$.

The above theorem is much stronger than the main result in [U2] where only the automorphisms linear with respect to $x$ and $y$ are dealt.

An important partial case of Example 2.15 gives a large class of wild automorphisms and wild coordinates. (Recall that Corollary 2.16 gives only the $z$-wildness of the automorphisms.)

Example 2.31. (Drensky and Yu [DY4, DY5]) Let $h(t, z) \in K\langle t, z\rangle$ and let $h(0,0)=0$. If the linear component (with respect to $x, y$ ) $h_{1}(x z-z y, z)$ of $h(x z-z y, z)$ is not equal to 0 , then the automorphism

$$
\sigma_{h}=(x+z h(x z-z y, z), y+h(x z-z y, z) z, z)
$$

belongs to the class of wild automorphisms in Theorem [2.29]

Example 2.32. (Drensky and Yu [DY4, DY5]) A minor modification of the Anick automorphism is the automorphism of $K\langle x, y, z\rangle$

$$
\omega_{m}=\left(x+z(x z-z y)^{m}, y+(x z-z y)^{m} z, z\right) .
$$

Note that the automorphisms $\omega_{m}, m>1$, are not covered by Theorem 2.29, as the polynomials $z(x z-z y)^{m}$ and $(x z-z y)^{m} z$ have no linear components with respect to $x$ and $y$.

Theorem 2.33. (Drensky and Yu [DY4, DY5]) The above automorphisms $\omega_{m}$ are wild for all $m \geq 1$.

Problem 2.34. Are the two nontrivial coordinates of the above automorphism $\omega_{m}, m>1$, both wild?

The most general form of the result of Umirbaev U2 gives that the automorphism $\vartheta=(f, g, h)$ of the free metabelian algebra $M(x, y, z)$ is wild, if it induces the identity automorphism of $K[x, y, z]$ and the matrix $J_{2}(\vartheta)\left(z_{1}, z_{2}\right)$ cannot be presented as a product of elementary matrices with entries from $K\left[z_{1}, z_{2}\right]$, see Theorem 2.21. Hence the classes of wild automorphisms and wild coordinates in Theorem 2.29. Example 2.31 and Example 2.32 are not covered by Umirbaev U2].

Now we are going to show that at least two coordinates of the automorphisms of the class of Umirbaev are wild. 
Theorem 2.35. (Drensky and Yu DY4, DY5]) Let $\vartheta=(f, g, h)$ be an automorphism of the free metabelian algebra $M(x, y, z)$ which induces the identity automorphism of $K[x, y, z]$ and the matrix $J_{2}(\vartheta)\left(z_{1}, z_{2}\right)$ does not belong to $G E_{2}\left(K\left[z_{1}, z_{2}\right]\right)$. Then the two coordinates $f(x, y, z)$ and $g(x, y, z)$ are both wild.

The above results suggest the following problems.

Problem 2.36. Is it true that the two nontrivial coordinates of a wild automorphism of $K\langle x, y, z\rangle$ fixing $z$ are both wild?

Problem 2.37. Is it true that every wild automorphism of $K\langle x, y, z\rangle$ contains at least two wild coordinates?

Finally, we want to mention that the free metabelian algebra $C$ has quite peculiar wild automorphisms which cannot be lifted to automorphisms of $K\langle x, y, z\rangle$. For example, such an automorphism is

$$
\rho=\left(x+x^{2}[y, z], y, z\right) .
$$

Note that its Jacobian matrix

$$
J_{M}(\tau)=\left(\begin{array}{ccc}
1 & 0 & 0 \\
x_{1}^{2}\left(z_{2}-z_{1}\right) & 1 & 0 \\
x_{1}^{2}\left(y_{1}-y_{2}\right) & 0 & 1
\end{array}\right)
$$

is a product of two elementary matrices. We refer to [DY5] for details.

Problem 2.38. Is the polynomial $x+x^{2}[y, z]$ a wild coordinate of $C$ ? Can it be lifted to a coordinate of $K\langle x, y, z\rangle$ ?

Problem 2.39. Do there exist wild automorphisms and wild coordinates of the free metabelian algebra $C_{n}$ of rank $n>3$ ? Are there wild automorphisms similar to the automorphism $\rho$ constructed above?

\section{Open Problems and Conjectures}

For some of the problems below we need char $K=0$. For simplicity of the exposition, we consider only the case of characteristic 0. Also, when we discuss algorithmic problems, we assume that the base field is constructive. We fix a finite set $X=\left\{x_{1}, \ldots, x_{n}\right\}$. Some of the problems, considered for $X=\{x, y, z\}$ have two versions, depending whether we assume that the automorphisms fix $z$ (or the derivations annihilate $z$ ).

Yagzhev [Y] suggested an algorithm which determines whether an endomorphism of $K\langle X\rangle$ is an automorphism. Hence we can check 
which $z$-endomorphisms of $K\langle x, y, z\rangle$ are automorphisms. Also, we have seen how to recognize the $z$-tame automorphisms and coordinates of $K\langle x, y, z\rangle$. In the view of the results in [SY1, DY2, SU1, SU3] exposed in the survey part of the present paper, we suggest:

Problem 3.1. Find algorithms which decide whether a given polynomial in $K\langle x, y, z\rangle$ is a coordinate for a z-wild automorphism.

Conjecture 3.2. If $\varphi$ is a wild z-automorphism of $K[x, y, z]$, then it cannot be lifted to a z-automorphism (to any automorphism) of $K\langle x, y, z\rangle$.

By the theorem of Umirbaev [U1], every automorphism of the polynomial algebra $K[X]$ can be lifted to an automorphism of the free metabelian algebra $M(X)=K\langle X\rangle / C^{2}$, where $C$ is the commutator ideal of $K\langle X\rangle$. A concrete lifting (which also preserves $z$ ) of the Nagata automorphism from $K[x, y, z]$ to $M(x, y, z)$ was given by the authors and Gutierrez DGY]. It follows easily from the theory of finitely generated PI-algebras that the theorem of Umirbaev holds also for $K\langle X\rangle / T(R)$, where $T(R)$ is the T-ideal of the polynomial identities (depending on the finite number of variables $X$ ) of a PI-algebra $R$ which satisfies a nonmatrix polynomial identity. The same lifting of the Nagata automorphism to $M(x, y, z)$ can be used as a lifting to $K\langle x, y, z\rangle / T(R)$.

Problem 3.3. Describe the (z-)automorphisms of $K[x, y, z]$ which can be lifted to automorphisms of $K\langle x, y, z\rangle / T\left(M_{n}(K)\right)$ and $K\langle x, y, z\rangle / T^{2}\left(M_{n}(K)\right)$, where $T\left(M_{n}(K)\right)$ is the set of all polynomial identities of the $n \times n$ matrix algebra. If all (z-)automorphisms of $K[x, y, z]$ can be lifted to $K\langle x, y, z\rangle / T^{2}\left(M_{n}(K)\right)$, then they can be lifted also to (z-)automorphisms of any relatively free algebra $K\langle x, y, z\rangle / T(R)$. The case $K\langle x, y, z\rangle / T\left(M_{2}(K)\right)$ is especially interesting because the structure of the algebra $K\langle X\rangle / T\left(M_{2}(K)\right)$ is well understood and one knows how to work effectively there.

In the case of the polynomial algebra $K[X]$, if $\delta$ is a locally nilpotent derivation and $K[X]^{\delta}$ is the algebra of constants (i.e., the kernel) of $\delta$, then for any $w \in K[X]^{\delta}$, the mapping $\Delta=w \delta$ is again a locally nilpotent derivation of $K[X]$ with the same algebra of constants as $\delta$. This fact is used to construct new automorphisms of $K[X]$, including the Nagata automorphism and to prove that some classes of automorphisms are stably tame, see the paper by Smith $\mathbf{S m}$, for better understanding of the automorphisms of $K[X]$, see the book by van 
den Essen E2, in the proof of Makar-Limanov of the tameness of the automorphisms of $K[x, y]$, see [ML3] or the book by one of the authors [D], etc. This construction does not produce anymore locally nilpotent derivations in $K\langle X\rangle$ because for the proof that $\Delta=w \delta$ is a derivation one needs that $w$ is in the centre of the algebra.

Problem 3.4. Starting from a locally nilpotent derivation $\delta$ of $K\langle X\rangle$, find constructions which produce other locally nilpotent derivations, preferable with the same kernel as $\delta$. Can every locally nilpotent derivation of $K[X]$ be lifted to a locally nilpotent derivation of $K\langle X\rangle$ ?

Problem 3.5. Can every locally nilpotent derivation of $K[X]$ be lifted to a locally nilpotent derivation of $K\langle X\rangle / T(R)$, where $R$ is a PIalgebra? Can the derivation $\Delta=\left(y^{2}+x z\right) \delta, \delta:(x, y, z) \rightarrow(-2 y, z, 0)$, (which is crucial for the stable tameness of the Nagata automorphism) be lifted to a locally nilpotent derivation of the free metabelian algebra $M_{3}(x, y, z)$ ?

Recently van den Essen and Peretz [EP] studied the automorphisms $\varphi$ of $K[X]$ such that $\varphi\left(x_{j}\right)=\sum_{i=1}^{n} w_{i j} x_{i}$, where each $w_{i j}$ is $\varphi$-invariant, i.e., these automorphisms are "linear" with coefficients from the algebra $K[X]^{\varphi}$ of fixed points. They related them with locally nilpotent derivations.

Problem 3.6. Describe, in the spirit of $[\mathrm{EP}]$, the automorphisms $\varphi$ of $K\langle X\rangle$ with the property that

$$
\varphi\left(x_{j}\right)=\sum_{i=1}^{n} \sum_{p} a_{i j p} x_{i} b_{i j p}, \quad a_{i j p}, b_{i j p} \in K\langle X\rangle^{\varphi} .
$$

In the commutative case Lamy [L1, L2] described the "nonaffine orthogonal group", i.e., the group of automorphisms of $K[x, y, z]$ which fixes a nondegenerate quadratic form. Considering the form $y^{2}+x z$, he proved that the group contains automorphisms which do not belong to the subgroup generated by Nagata-like automorphisms. Recall also the general construction due to Freudenburg [F1, F2] of locally nilpotent derivations of $K[x, y, z]$ which do not annulate any coordinate. Among them are derivations annulating the form $y^{2}+x z$.

Problem 3.7. Describe the automorphisms of $K\langle x, y, z\rangle$ which fix the form $2 y^{2}+x z+z x$. Describe the automorphisms with this property which fix also $z$. 


\section{REFERENCES}

[AL] J. Alev, L. Le Bruyn, Automorphisms of generic 2 by 2 matrices, in "Perspectives in Ring Theory (Antwerp, 1987)", NATO Adv. Sci. Inst. Ser. C Math. Phys. Sci. 233, Kluwer Acad. Publ., Dordrecht, 1988, 69-83.

[Ba] H. Bass, A non-triangular action of $\mathbb{G}_{a}$ on $\mathbb{A}^{3}$, J. Pure Appl. Algebra 33 (1984), 1-5.

[Be] G.M. Bergman, Wild automorphisms of free P.I. algebras, and some new identities, preprint.

[B1] J. Berson, Stably tame coordinates, J. Pure Appl. Algebra 170 (2002), No. 2-3, 131-143.

[B2] J. Berson, Two-dimensional stable tameness over Noetherian domains of dimension one, J. Pure Appl. Algebra 178 (2003), No. 2, 115-129.

[BE] J. Berson, A. van den Essen, An algorithm to find a coordinate's mate, J. Symbolic Comput. 36 (2003), No. 6, 835-843.

[CK] J. Chądzyński, T. Krasiński, On the Łojasiewicz exponent at infinity for polynomial mappings of $C^{2}$ into $C^{2}$ and components of polynomial automorphisms of $C^{2}$, Ann. Polon. Math. 57 (1992), No. 3, 291-302.

[C1] P.M. Cohn, On the structure of the $\mathrm{GL}_{2}$ of a ring, Inst. Hautes Études Sci. Publ. Math. 30 (1966), 5-53.

[C2] P.M. Cohn, Free Rings and Their Relations, Second Edition, Acad. Press, 1985.

[Cz] A.J. Czerniakiewicz, Automorphisms of a free associative algebra of rank 2. I, II, Trans. Amer. Math. Soc. 160 (1971), 393-401; 171 (1972), 309-315.

[DF] D. Daigle, G. Freudenburg, Locally nilpotent derivations over a UFD and an application to rank two locally nilpotent derivations of $k\left[X_{1}, \ldots, X_{n}\right], \mathrm{J}$. Algebra 204 (1998), 353-371.

[Di] W. Dicks, A commutator test for two elements to generate the free algebra of rank two, Bull. London Math. Soc. 14 (1982), No. 1, 48-51.

[DiL] W. Dicks, J. Lewin, A Jacobian conjecture for free associative algebras, Comm. Algebra 10 (1982), 1285-1306.

[DN] The Dniester Notebook, Unsolved Problems in the Theory of Rings and Modules (Russian), Fourth edition, Compiled by V.T. Filippov, V.K. Kharchenko, and I.P. Shestakov, Novosibirsk, 1993.

[D] V. Drensky, Free Algebras and PI-Algebras, Springer-Verlag, Singapore, 1999.

[DES] V. Drensky, A. van den Essen, D. Stefanov, New stably tame automorphisms of polynomial algebras, J. Algebra 226 (2000), 629-638.

[DG1] V. Drensky, C.K. Gupta, New automorphisms of generic matrix algebras and polynomial algebras, J. Algebra 194 (1997), 408-414.

[DG2] V. Drensky, C.K. Gupta, Constants of Weitzenböck derivations and invariants of unipotent transformations acting on relatively free algebras, J. Algebra 292 (2005), 393-428.

[DGY] V. Drensky, J. Gutierrez, J.-T. Yu, Gröbner bases and the Nagata automorphism, J. Pure Appl. Algebra 135 (1999), 135-153.

[DY1] V. Drensky, J.-T. Yu, Automorphisms and coordinates of polynomial algebras, in "Combinatorial and Computational Algebra (Hong Kong, 1999)"; Eds. K.Y. Chan, A.A. Mikhalev, M.-K. Siu, J.-T. Yu, and E. Zelmanov, Contemp. Math. 264, 2000, 179-206. 
[DY2] V. Drensky, J.-T. Yu, Tame and wild coordinates of $K[z][x, y]$, Trans. Amer. Math. Soc. 353 (2001), No. 2, 519-537.

[DY3] V. Drensky, J.-T. Yu, Automorphisms fixing a variable of $K\langle x, y, z\rangle$, J. Algebra 291 (2005), No. 1, 250-258.

[DY4] V. Drensky, J.-T. Yu, The strong Anick conjecture, Proc. Nat. Acad. Sci., 103 (2006), No. 13, 4836-4840.

[DY5] V. Drensky, J.-T. Yu, The strong Anick conjecture is true, preprint.

[DY6] V. Drensky, J.-T. Yu, Automorphic equivalence problem for free associative algebras of rank two, International J. Algebra and Computations (to appear).

[DY7] V. Drensky, J.-T. Yu, Tame automorphisms fixing a variable of free associative algebras of rank three, preprint.

[Ed] E. Edo, Totally stably tame variables, J. Algebra 287 (2005), No. 1, 15-31.

[EdV] E. Edo, S. Vénéreau, Length 2 variables of $A[x, y]$ and transfer, in "Polynomial Automorphisms and Related Topics (Kraków, 1999)", Ann. Polon. Math. 76 (2001), No. 1-2, 67-76.

[E1] A. van den Essen, Locally nilpotent derivations and their applications. III, J. Pure Appl. Algebra 98 (1995), No. 1, 15-23.

[E2] A. van den Essen, Polynomial Automorphisms and the Jacobian Conjecture, Birkhäuser, Progress in Mathematics 190, Basel-Boston, 2000.

[E3] A. van den Essen, The solution of the tame generators conjecture according to Shestakov and Umirbaev, Colloq. Math. 100 (2004), No. 2, 181-194.

[EP] A. van den Essen, R. Peretz, Polynomial automorphisms and invariants, J. Algebra 269 (2003), 317-328.

[Fo] E. Formanek, Central polynomials for matrix rings, J. Algebra 23 (1972), 129-132.

[F1] G. Freudenburg, Local slice constructions in $k[X, Y, Z]$, Osaka J. Math. 34 (1997) 757-767.

[F2] G. Freudenburg, Actions of $G_{a}$ on $A^{3}$ defined by homogeneous derivations, J. Pure Appl. Algebra 126 (1998), 169-181.

[J] H.W.E. Jung, Über ganze birationale Transformationen der Ebene, J. Reine und Angew. Math. 184 (1942), 161-174.

[K] W. van der Kulk, On polynomial rings in two variables, Nieuw Archief voor Wiskunde (3) 1 (1953), 33-41.

[L1] S. Lamy, Les automorphismes polynomiaux de $C^{3}$ préservant une forme quadratique, C. R. Acad. Sci. Paris Sér. I Math. 328 (1999), 883-886.

[L2] S. Lamy, Automorphismes polynomiaux préservant une action de groupe, Bol. Soc. Mat. Mexicana (3) 9 (2003), No. 1, 1-19.

[ML1] L.G. Makar-Limanov, On automorphisms of free algebra with two generators (Russian), Funk. Analiz i ego Prilozh. 4 (1970), No. 3, 107-108. Translation: Functional Anal. Appl. 4 (1970), 262-263.

[ML2] L.G. Makar-Limanov, On Automorphisms of Certain Algebras (Russian), Ph.D. Thesis, Moscow, 1970.

[ML3] L.G. Makar-Limanov, Automorphisms of polynomial ring - a shortcut, preprint.

[MLSY] L. Makar-Limanov, V. Shpilrain, J.-T. Yu, Equivalence of polynomials under automorphisms of $K[x, y]$, J. Pure Appl. Algebra (to appear). 
[MSY] A.A. Mikhalev, V. Shpilrain, J.-T. Yu, Combinatorial Methods. Free Groups, Polynomials, Free Algebras, CMS Books in Mathematics, Springer, New York, 2004.

[N] M. Nagata, On the Automorphism Group of $k[x, y]$, Lect. in Math., Kyoto Univ., Kinokuniya, Tokyo, 1972.

[P] H. Park, A realization algorithm for $\mathrm{SL}_{2}\left(R\left[x_{1}, \cdots, x_{m}\right]\right)$ over the Euclidean domain, SIAM J. Matrix Anal. Appl. 21 (1999), No. 1, 178-184.

[PW] H. Park, C. Woodburn, An algorithmic proof of Suslin's stability theorem for polynomial rings, J. Algebra 178 (1995), 277-298.

[Sc] A.N. Schofield, Representation of Rings over Skew Fields, London Mathematical Society Lecture Note Series, 92, Cambridge University Press, Cambridge, 1985.

[SU1] I.P. Shestakov, U.U. Umirbaev, The Nagata automorphism is wild, Proc. Nat. Acad. Sci. 100 (2003), No. 22, 12561-12563.

[SU2] I.P. Shestakov, U.U. Umirbaev, Poisson brackets and two-generated subalgebras of rings of polynomials, J. Amer. Math. Soc. 17 (2004), 181-196.

[SU3] I.P. Shestakov, U.U. Umirbaev, The tame and the wild automorphisms of polynomial rings in three variables, J. Amer. Math. Soc. 17 (2004), 197-227.

[SY1] V. Shpilrain, J.-T. Yu, Polynomial automorphisms and Gröbner reductions, J. Algebra 197 (1997), 546-558.

[SY2] V. Shpilrain, J.-T. Yu, On generators of polynomial algebras in two commuting or non-commuting variables, J. Pure Appl. Algebra 132 (1998), 309-315.

[SY3] V. Shpilrain, J.-T. Yu, Embeddings of curves in the plane, J. Algebra 217 (1999), 668-678.

[Sm] M. K. Smith, Stably tame automorphisms, J. Pure Appl. Algebra 58 (1989), 209-212.

[Su A.A. Suslin, On the structure of the special linear group over polynomial rings (Russian), Izv. Akad. Nauk SSSR Ser. Mat. 41 (1977), 235-252. Translation: Math. USSR Izv. 11 (1977), 221-239.

[THK] L. Tolhuizen, H. Hollmann, T.A.C.M. Kalker, On the realizability of biorthogonal, $m$-dimensional two-band filter banks, IEEE Trans. Signal Processing 43 (1995), No. 3, 640-648.

[U1] U.U. Umirbaev, On the extension of automorphisms of polynomial rings (Russian), Sibirsk. Mat. Zh. 36 (1995), 911-916. Translation: Sib. Math. J. 36 (1995), 787-791.

[U2] U.U. Umirbaev, Tame and wild automorphisms of polynomial algebras and free associative algebras, Max-Planck-Institute for Mathematics, Bonn, Preprint MPIM2004-108, J. Algebra (to appear).

[UY] U.U. Umirbaev, J.-T. Yu, The strong Nagata conjecture, Proc. Nat. Acad. Sci. 101 (2004), No. 13, 4352-4355.

[Wi] P.G. Wightwick, Equivalence of polynomials under automorphisms of $\mathbb{C}^{2}, \mathrm{~J}$. Pure Appl. Algebra 157 (2001), No. 2-3, 341-367.

[Wr] D. Wright, The amalgamated free product structure of $G L_{2}\left(k\left[X_{1}, \ldots, X_{n}\right]\right)$ and the weak Jacobian theorem for two variables, J. Pure Appl. Algebra 12 (1978), 235-251. 
[Y] A.V. Yagzhev, The algorithmic problem of recognizing automorphisms among the endomorphisms of free associative algebras of finite rank (Russian), Sib. Mat. Zh. 21 (1980), 193-199. Translation: Sib. Math. J. 21 (1980), 142-146.

Institute of Mathematics and Informatics, Bulgarian Academy of Sciences, 1113 Sofia, Bulgaria

E-mail address: drensky@math.bas.bg

Department of Mathematics, the University of Hong Kong, Hong KONG SAR, ChINA

E-mail address: yujt@hkucc.hku.hk 